\title{
PRODUTOS MULTIPLAMENTE TORCIDOS
}

\author{
Emílio Pedro Gueuvoghlanian
}

DISSERTAÇÃO APRESENTADA

$\mathrm{AO}$

INSTITUTO DE MATEMÁTICA E ESTATÍSTICA

DA

UNIVERSIDADE DE SÃO PAULO

PARA

OBTENÇÃO DO TÍTULO DE MESTRE

$\mathrm{EM}$

CIÊNCIAS

Área de Concentração: Matemática

Orientadora: Profa. Dra. Rosa Maria dos Santos Barreiro Chaves

Durante a elaboração deste trabalho o autor recebeu auxílio financeiro do CNPq.

São Paulo, agosto de 2006. 
PRODUTOS MULTIPLAMENTE TORCIDOS

Este exemplar corresponde à redação final da dissertação devidamente corrigida e defendida por Emílio Pedro Gueuvoghlanian e aprovada pela Comissão Julgadora.

São Paulo, agosto de 2006.

\section{Banca Examinadora:}

- Profa. Dra. Rosa Maria dos Santos Barreiro Chaves (Orientadora) - IME-USP

- Prof. Dr. Antonio Carlos Asperti - IME-USP

- Prof. Dr. Guillermo Antonio Lobos - DM-UFSCar 


\section{Agradecimentos}

Desejo agradecer à Profa. Dra. Rosa Maria dos Santos Barreiro Chaves, pela orientação e ao CNPq, pelo auxílio financeiro. 


\section{Resumo}

Neste trabalho, produtos multiplamente torcidos são definidos. Em casos particulares, relevantes à teoria da relatividade geral, analisam-se sob quais condições as variedades correspondentes são variedades de Einstein ou possuem curvatura escalar constante. 


\section{Abstract}

In this work, multiply warped products are defined. In particular cases, which are relevant to the theory of general relativity, conditions under which the related manifolds are Einstein manifolds or have constant scalar curvature are analyzed. 


\section{Sumário}

Introdução

$\begin{array}{lr}1 \text { Produtos multiplamente torcidos } & 7\end{array}$

2 Espaços-tempo de Robertson-Walker generalizados 25

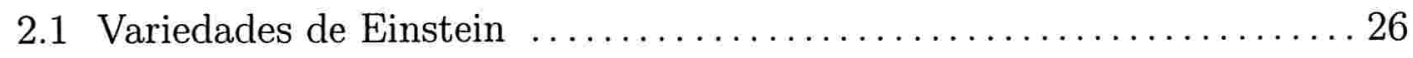

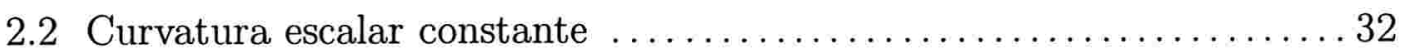

3 Espaços-tempo de Kasner generalizados $\quad 37$

3.1 Espaços-tempo de dimensão arbitrária $\ldots \ldots \ldots \ldots \ldots \ldots \ldots \ldots \ldots \ldots \ldots \ldots \ldots$

3.2 Espaços-tempo de dimensão quatro $\ldots \ldots \ldots \ldots \ldots \ldots \ldots \ldots \ldots \ldots \ldots \ldots \ldots$

3.3 Espaços-tempo de Kasner do tipo II $\ldots \ldots \ldots \ldots \ldots \ldots \ldots \ldots \ldots$

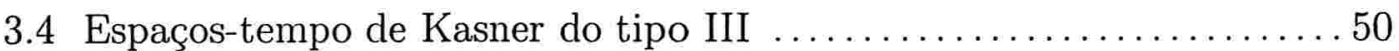

4 Espaços-tempo BTZ generalizados $\quad 55$

$\begin{array}{ll}\text { Apêndice } & 61\end{array}$

$\begin{array}{lr}\text { Referências bibliográficas } & 69\end{array}$ 


\section{Introdução}

O contexto ao qual esta dissertação pertence é o da geometria semi-Riemanniana [22], ou seja, o estudo das variedades diferenciáveis munidas de um tensor métrico de assinatura arbitrária. A geometria Riemanniana, que corresponde a uma métrica positiva definida e a geometria de Lorentz, que corresponde a uma métrica com índice igual a um, são casos particulares.

O conceito de produto torcido é uma generalização do conceito de variedade produto (munida da métrica usual) [8] e [22]. A estrutura de variedade diferenciável corresponde à variedade produto usual. Entretanto, a métrica é definida de um modo bem particular:

Definição I.1 Sejam $\left(B, g_{B}\right)$ e $\left(F, g_{F}\right)$ duas variedades semi-Riemannianas, onde $g_{B}$ e $g_{F}$ indicam as métricas correspondentes e seja $b: B \rightarrow(0, \infty)$ uma função de classe $C^{\infty}$. Então, denomina-se produto torcido $B \times{ }_{b} F$ à variedade produto $B \times F$ munida do tensor métrico definido por

$$
g=\pi^{*}\left(g_{B}\right) \oplus(b \circ \pi)^{2} \sigma^{*}\left(g_{F}\right)
$$

onde $\pi: B \times F \rightarrow B$ e $\sigma: B \times F \rightarrow F$ são as projeções canônicas e * representa o "pull-back" de tensores.

A geometria de Lorentz [5] encontra como sua principal aplicação e motivação para o seu desenvolvimento a teoria da relatividade geral [21]. Historicamente, 
tendo como ponto de partida conceitual a teoria da relatividade restrita, esta teoria foi desenvolvida com o objetivo de incorporar uma das interações fundamentais conhecidas em física, a gravitação. Tal teoria, com ampla verificação experimental, encontra seu uso em astrofísica e cosmologia.

As equações de Einstein desempenham um importante papel na teoria da relatividade geral. Com o intuito de explicar a relação entre estas equações e o objeto de estudo desta dissertação, apresentam-se, agora, alguns conceitos. Entende-se por $(M, g, D)$ uma variedade diferenciável $M$ munida de um tensor métrico $g$ e uma conexão de Levi-Civita $D$. Uma constante cosmológica é uma função constante $\Lambda: M \rightarrow \mathbb{R}$. Um tensor de energia-momento $T$ é um tensor covariante simétrico de ordem $(0,2)$ definido em $M$. Do ponto de vista físico, algumas condições particulares, descritas em detalhes no livro de Hawking e Ellis [15], devem ser impostas sobre este tensor. Não é necessário nesta dissertação discorrer sobre estas condições. Aqui, apenas menciona-se que as condições mínimas a serem impostas, segundo Hawking e Ellis, correspondem ao postulado da causalidade local, à condição de divergência nula e à condição fraca sobre a energia. Veja a referência [15] para uma formulação precisa destas condições. Considere a seguinte definição, conforme as referências [15] e [23]:

Definição I.2 $((M, g, D), T, \Lambda)$ é uma solução das equações de Einstein se

$$
\text { Ric }-\frac{1}{2} S g+\Lambda g=8 \pi T
$$

onde Ric e $S$ são, respectivamente, o tensor de Ricci e a curvatura escalar relativos à conexão $D$.

Uma classificação das soluções conhecidas das equações de Einstein pode ser encontrada na referência [20]. 
A conexão entre produtos torcidos e relatividade geral está no fato de que foi verificado que variedades diferenciáveis $(M, g, D)$ correspondentes a soluções conhecidas das equações de Einstein $((M, g, D), T, \Lambda)$ podiam ser expressas como produtos torcidos [4] e [22]. Posteriormente, produtos torcidos foram utilizados com o intuito de obter novas soluções dessas equações [5].

Recentemente, generalizações do conceito de produto torcido foram introduzidas [2], [6], [26] e [27]. Nesta dissertação, restringe-se a discussão aos produtos multiplamente torcidos [26], cuja definição é a que segue:

Definição I.3 Sejam $\left(B, g_{B}\right)$ e $\left(F_{i}, g_{F_{i}}\right)$ variedades semi-Riemannianas e sejam $b_{i}$ : $B \rightarrow(0, \infty)$ funções de classe $C^{\infty}, \forall i \in\{1,2, \ldots, m\}$, onde $m \in \mathbb{Z}, m>0$. Um produto multiplamente torcido é uma variedade produto $M=B \times F_{1} \times \cdots \times F_{m}$ munida de uma métrica definida por:

$$
g=\pi^{*}\left(g_{B}\right) \oplus\left(b_{1} \circ \pi\right)^{2} \sigma_{1}^{*}\left(g_{F_{1}}\right) \oplus \cdots \oplus\left(b_{m} \circ \pi\right)^{2} \sigma_{m}^{*}\left(g_{F_{m}}\right),
$$

onde $\pi: M \rightarrow B$ e $\sigma_{i}: M \rightarrow F_{i}$ são as projeções canônicas. Cada variedade $\left(F_{i}, g_{F_{i}}\right)$ é denominada fibra e a variedade $\left(B, g_{B}\right)$ é denominada base. Denota-se $M=B \times{ }_{b_{1}} F_{1} \times \cdots \times{ }_{b_{m}} F_{m}$.

Do ponto de vista da geometria semi-Riemanniana, os pré-requisitos para o desenvolvimento deste projeto correspondem aos capítulos iniciais do livro de B. O'Neill, "Semi-Riemannian Geometry" [22]. Os capítulos do mesmo livro dedicados à teoria da relatividade geral constituem uma introdução concisa ao assunto. O conteúdo deste projeto é fortemente baseado no artigo "Curvature of multiply warped products", de F. Dobarro e B. Ünal [13]. O conteúdo desta dissertação é o que segue:

No Capítulo 1, os principais resultados obtidos referem-se à relação entre a derivada covariante, o tensor de Riemann, o tensor de Ricci e a curvatura escalar da 
variedade correspondente ao produto torcido e os mesmos objetos definidos na base e em cada uma das fibras [2], [10], [11], [13], [22], [25] e [26].

A partir do Capítulo 2, toda a discussão restringe-se ao caso particular dos espaços-tempo de Robertson-Walker generalizados [13] e [24]. Tais espaços-tempo são uma extensão do espaço-tempo de Robertson-Walker [22] usual, um dos modelos básicos em cosmologia.

Definição I.4 Um espaço-tempo de Robertson-Walker generalizado é um produto multiplamente torcido tal que a base $B$ é um intervalo conexo aberto $I$ da forma $I=\left(t_{1}, t_{2}\right)$ munido da métrica definida negativa $g_{B}=-d t \otimes d t, \forall t \in I$, onde $-\infty \leq t_{1}<t_{2} \leq \infty$ e cada fibra $\left(F_{i}, g_{F_{i}}\right)$ é Riemanniana, $\forall i \in\{1, \ldots, m\}$.

Um conceito importante é o de variedade de Einstein [7]. Uma variedade $(M, g)$ semi-Riemanniana é dita de Einstein se existe uma função $\lambda: M \rightarrow \mathbb{R}$ de classe $C^{\infty}$ tal que Ric $=\lambda g$. Algumas condições necessárias e/ou suficientes para que um espaço-tempo de Robertson-Walker generalizado possua curvatura escalar constante ou seja uma variedade de Einstein são consideradas [13]:

Teorema I.5 Seja $M$ um espaço-tempo de Robertson-Walker generalizado. Então, $M$ é uma variedade de Einstein com tensor de Ricci $\lambda g$ se e somente se as seguintes condições são satisfeitas $\forall i \in\{1, \ldots, m\}$ :

(1) Cada fibra $\left(F_{i}, g_{F_{i}}\right)$ é uma variedade de Einstein com tensor de Ricci $\lambda_{F_{i}} g_{F_{i}}$,

(2) $\sum_{i=1}^{m} s_{i} \frac{b_{i}^{\prime \prime}}{b_{i}}=\lambda e$

(3) $\lambda_{F_{i}}+b_{i} b_{i}^{\prime \prime}+\left(s_{i}-1\right)\left(b_{i}^{\prime}\right)^{2}+b_{i} b_{i}^{\prime} \sum_{k=1, k \neq i}^{m} s_{k} \frac{b_{k}^{\prime}}{b_{k}}=\lambda b_{i}^{2}$,

onde $s_{i}$ é a dimensão de $F_{i}$. 
Proposição I.6 Seja M um espaço-tempo de Robertson-Walker generalizado. Se $M$ tem curvatura escalar constante $S$, então, cada fibra $F_{i}$ tem curvatura escalar constante $S_{F_{i}}, \forall i \in\{1, \ldots, m\}$.

Nos Capítulos 3 e 4 são consideradas condições necessárias e/ou suficientes para que casos particulares do espaço-tempo de Robertson-Walker generalizado possuam curvatura escalar constante ou sejam variedades de Einstein. Segue, aqui, uma descrição breve destes casos:

No Capítulo 3, abordam-se os espaços-tempo de Kasner generalizados [13]. O espaço-tempo de Kasner usual [18] data dos primórdios da teoria da relatividade geral, como um exemplo de cosmologia anisotrópica [21]. Recentemente, diferentes generalizações do espaço-tempo de Kasner usual foram introduzidas, no espírito das teorias de Kaluza-Klein [17] e [19]. Ou seja, teorias com a dimensão do espaço-tempo maior do que quatro relevantes à física das partículas elementares.

Ainda no Capítulo 3, os espaços-tempo de Kasner generalizados de dimensão quatro são considerados em detalhes. Este caso particular é bastante relevante, pois, na medida em que efeitos quânticos puderem ser ignorados em cosmologia, o espaço-tempo é descrito através de uma variedade de dimensão quatro [21].

No Capítulo 4, os espaços-tempo BTZ (Bañados, Teitelboim e Zanelli) generalizados de dimensão $(2+1)$ são analisados [13]. O espaço-tempo tridimensional BTZ clássico exibe as principais características de um buraco negro, com a vantagem de ser mais simples do que os modelos quadridimensionais [3].

No Apêndice, são apresentados conceitos básicos da geometria semi-Riemanniana [22], necessários ao desenvolvimento do trabalho.

No que concerne a desenvolvimentos futuros dos temas relativos a produtos multiplamente torcidos, uma importante linha de pesquisa pode ser encontrada na referência [9]. 


\section{Capítulo 1}

\section{Produtos multiplamente torcidos}

Definição 1.1 Sejam $\left(B, g_{B}\right)$ e $\left(F_{i}, g_{F_{i}}\right)$ variedades semi-Riemannianas e sejam $b_{i}$ : $B \rightarrow(0, \infty)$ funções de classe $C^{\infty}, \forall i \in\{1,2, \ldots, m\}$, onde $m \in \mathbb{Z}, m>0$. Denomina-se produto multiplamente torcido à variedade produto $M=B \times F_{1} \times$ $\cdots \times F_{m}$ munida da métrica definida por:

$$
g=\pi^{*}\left(g_{B}\right) \oplus\left(b_{1} \circ \pi\right)^{2} \sigma_{1}^{*}\left(g_{F_{1}}\right) \oplus \cdots \oplus\left(b_{m} \circ \pi\right)^{2} \sigma_{m}^{*}\left(g_{F_{m}}\right),
$$

onde $\pi: M \rightarrow B$ e $\sigma_{i}: M \rightarrow F_{i}$ são as projeções canônicas e $*$ representa o "pull-back" de tensores.

Cada variedade $\left(F_{i}, g_{F_{i}}\right)$ é denominada fibra e a variedade $\left(B, g_{B}\right)$ é denominada base. Denota-se

$$
M:=B \times{ }_{b_{1}} F_{1} \times \cdots \times{ }_{b_{m}} F_{m} .
$$

Os resultados apresentados nesta seção, assim como suas demonstrações, para o caso $m=1$, podem ser encontrados no livro de O'Neill [22]. Segundo consta na referência [26], os resultados equivalentes para $m=2$ constam da tese de Baker [2]. Resultados para um valor de $m$ arbitrário podem ser encontrados sem demonstração nos artigos de Dobarro e Ünal [26] e [13]. No artigo de Choi [10], onde consideram-se métricas que não são necessariamente de classe $C^{\infty}$, apenas os termos extras que são 
decorrentes deste fato são justificados. No artigo de Dobarro e Ünal [13] a referência à tese de Ünal pode ser encontrada [25].

Dado um produto multiplamente torcido $M=B \times{ }_{b_{1}} F_{1} \times \cdots \times{ }_{b_{m}} F_{m}$ e uma coleção de objetos definidos em $M, B$ e $\left\{F_{i}\right\}$, por exemplo, tensores, existem diferentes convenções encontradas na literatura quanto à notação utilizada. Aqui, segue-se a seguinte convenção: objetos definidos em $M$ não possuem índices enquanto que objetos definidos na base e nas fibras possuem índices. Por exemplo, $g, g_{B}$, representam, respectivamente, as métricas em $M$ e $B$. Em alguns casos, uma barra é colocada sobre o objeto para indicar levantamento. Por exemplo, $X=\bar{X}_{B}$ indica que o campo $X \in \mathscr{X}(M)$ é o levantamento de $X_{B} \in \mathscr{X}(B)$. Se em alguma parte deste trabalho uma convenção diferente for utilizada, o fato será explicitamente mencionado.

Nesta dissertação, todas as variedades são supostas conexas. O conjunto das funções de classe $C^{\infty}$ em uma variedade $M$ é denotado por $\mathcal{F}(M)$.

Proposição 1.2 Dados $M=B \times{ }_{b_{1}} F_{1} \times \cdots \times{ }_{b_{m}} F_{m}, \phi \in \mathcal{F}(B)$ e $\psi_{i} \in \mathcal{F}\left(F_{i}\right)$, então, $\forall i \in\{1, \ldots, m\}$,

$$
\begin{aligned}
\operatorname{grad}(\phi \circ \pi) & =\overline{\operatorname{grad}_{B} \phi} e \\
\operatorname{grad}\left(\psi_{i} \circ \sigma_{i}\right) & =\frac{\overline{\operatorname{grad}_{F_{i}} \psi_{i}}}{\left(b_{i} \circ \pi\right)^{2}}
\end{aligned}
$$

\section{Demonstração:}

(1.1) Deve-se mostrar que $\mathrm{d} \pi(\operatorname{grad}(\phi \circ \pi))=\operatorname{grad}_{B} \phi$ e $\mathrm{d} \sigma_{i}(\operatorname{grad}(\phi \circ \pi))=0, \forall i \in$ $\{1, \ldots, m\}$. Considere $p \in M, i \in\{1, \ldots, m\}$ e $V \in T_{p} F_{i}$ arbitrários. Então,

$$
g(\operatorname{grad}(\phi \circ \pi), V)=V(\phi \circ \pi)=\mathrm{d} \pi(V) \phi=0,
$$

pois $\mathrm{d} \pi(V)=0$. Logo, como

$$
g(\operatorname{grad}(\phi \circ \pi), V)=b_{i}^{2} g_{F_{i}}\left(\mathrm{~d} \sigma_{i}(\operatorname{grad}(\phi \circ \pi)), \mathrm{d} \sigma_{i}(V)\right)
$$


$V$ é arbitrário e $b_{i}: B \rightarrow(0, \infty)$, segue que

$$
\mathrm{d} \sigma_{i}(\operatorname{grad}(\phi \circ \pi))=0, \quad \forall i \in\{1, \ldots, m\} .
$$

Considere $p \in M$ e $X \in T_{p} B$ arbitrários. Então,

$$
\begin{aligned}
g_{B}(\mathrm{~d} \pi(\operatorname{grad}(\phi \circ \pi)), \mathrm{d} \pi(X)) & =g(\operatorname{grad}(\phi \circ \pi), X)=X(\phi \circ \pi) \\
& =\mathrm{d} \pi(X) \phi=g_{B}\left(\operatorname{grad}_{B} \phi, \mathrm{d} \pi(X)\right)
\end{aligned}
$$

Como $X$ é arbitrário, $\mathrm{d} \pi(\operatorname{grad}(\phi \circ \pi))=\operatorname{grad}_{B} \phi$.

(1.2) Deve-se mostrar que $\mathrm{d} \pi\left(\operatorname{grad}\left(\psi_{i} \circ \sigma_{i}\right)\right)=0, \mathrm{~d} \sigma_{j}\left(\operatorname{grad}\left(\psi_{i} \circ \sigma_{i}\right)\right)=0$, se $i \neq j \mathrm{e}$ $\left(b_{i} \circ \pi\right)^{2} \mathrm{~d} \sigma_{i}\left(\operatorname{grad}\left(\psi_{i} \circ \sigma_{i}\right)\right)=\left(\operatorname{grad}_{F_{i}} \psi_{i}\right) \circ \sigma_{i}$. Considere $p \in M$ e $X \in T_{p} B$ arbitrários. Então,

$$
\begin{aligned}
g_{B}\left(\mathrm{~d} \pi\left(\operatorname{grad}\left(\psi_{i} \circ \sigma_{i}\right)\right), \mathrm{d} \pi(X)\right) & =g\left(\operatorname{grad}\left(\psi_{i} \circ \sigma_{i}\right), X\right)=X\left(\psi_{i} \circ \sigma_{i}\right) \\
& =\mathrm{d} \sigma_{i}(X) \psi_{i}=0,
\end{aligned}
$$

pois $\mathrm{d} \sigma_{i}(X)=0$. Como $X$ é arbitrário, $\mathrm{d} \pi\left(\operatorname{grad}\left(\psi_{i} \circ \sigma_{i}\right)\right)=0$.

Considere $p \in M, j \in\{1, \ldots, m\}$ e $W \in T_{p} F_{j}$ arbitrários. Então,

$$
\begin{aligned}
\left(b_{j} \circ \pi\right)^{2} g_{F_{j}}\left(\mathrm{~d} \sigma_{j}\left(\operatorname{grad}\left(\psi_{i} \circ \sigma_{i}\right)\right), \mathrm{d} \sigma_{j}(W)\right) & =g\left(\operatorname{grad}\left(\psi_{i} \circ \sigma_{i}\right), W\right)=W\left(\psi_{i} \circ \sigma_{i}\right) \\
& =\left[\mathrm{d} \sigma_{i}(W) \psi_{i}\right] \circ \sigma_{i}=0, \text { se } i \neq j
\end{aligned}
$$

ou $\left[g_{F_{j}}\left(\operatorname{grad}_{F_{j}} \psi_{j}, \mathrm{~d} \sigma_{j}(W)\right)\right] \circ \sigma_{j}$, se $i=j$. Logo, como $W$ é arbitrário,

$$
\left(b_{j} \circ \pi\right)^{2} \mathrm{~d} \sigma_{j}\left(\operatorname{grad}\left(\psi_{j} \circ \sigma_{j}\right)\right)=\left(\operatorname{grad}_{F_{j}} \psi_{j}\right) \circ \sigma_{j} .
$$

Proposição 1.3 Dados $M=B \times{ }_{b_{1}} F_{1} \times \cdots \times{ }_{b_{m}} F_{m}, X, Y \in \mathcal{L}(B), V \in \mathcal{L}\left(F_{i}\right) e$ 
$W \in \mathcal{L}\left(F_{j}\right), i, j \in\{1, \ldots, m\}$, então

$$
\begin{aligned}
& D_{X} Y=\overline{D_{X_{B}}^{B} Y_{B}}, \\
& D_{X} V=D_{V} X=\frac{X\left(b_{i} \circ \pi\right)}{\left(b_{i} \circ \pi\right)} V e \\
& D_{V} W=\left\{\begin{array}{l}
0, \text { se } i \neq j, \quad e \\
\overline{D_{V_{F_{i}}}^{F_{i} W_{F_{i}}}-\frac{g(V, W)}{\left(b_{i} \circ \pi\right)} \operatorname{grad}\left(b_{i} \circ \pi\right), \text { se } i=j,}
\end{array}\right.
\end{aligned}
$$

onde $X=\overline{X_{B}}, Y=\overline{Y_{B}}, V=\overline{V_{F_{i}}}$ e $W=\overline{W_{F_{j}}}$.

\section{Demonstração:}

(1.3) Deve-se mostrar que $\mathrm{d} \pi\left(D_{X} Y\right)=D_{X_{B}}^{B} Y_{B}$ e $\mathrm{d} \sigma_{i}\left(D_{X} Y\right)=0, \forall i \in\{1, \ldots, m\}$.

Utilizando a fórmula de Koszul, resulta que $g\left(D_{X} Y, V\right)=0$. Analisando termo a termo: $g(X, V)=g(V, Y)=0$, pela definição da métrica. Pela Proposição A.1, $[Y, V]=[X, V]=0$. Pela mesma proposição, $[Y, X] \in \mathcal{L}(B)$. Logo, $g([Y, X], V)=0$. Além disso, $g(Y, X)$ é constante ao longo das fibras, logo, $V g(Y, X)=0$. Como $V$ é arbitrário, $\mathrm{d} \sigma_{i}\left(D_{X} Y\right)=0, \forall i \in\{1, \ldots, m\}$.

Dados $f_{i} \in F_{i}, \forall i \in\{1, \ldots, m\}$ fixos, mas arbitrários, $B \times f_{1} \times \cdots \times f_{m}$ é uma subvariedade de $M$. Considere $q \in B$ arbitrário, $p=\left(q, f_{1}, \cdots, f_{m}\right)$ e a restrição da projeção canônica $\pi: M \rightarrow B$ à subvariedade $B \times f_{1} \times \cdots \times f_{m}$, isto é, $\left.\pi\right|_{\left(B \times f_{1} \times \cdots \times f_{m}\right)} \rightarrow B$. Então, a restrição da métrica de $M$ à subvariedade $B \times f_{1} \times \cdots \times f_{m}$, dada pela aplicação

$$
g_{B \times f_{1} \times \cdots \times f_{m}}: T_{p}\left(B \times f_{1} \times \cdots \times f_{m}\right) \times T_{p}\left(B \times f_{1} \times \cdots \times f_{m}\right) \rightarrow \mathbb{R}
$$

definida por

$$
g_{B \times f_{1} \times \cdots \times f_{m}}\left(X_{p}, Y_{p}\right)=g_{B}\left(d\left(\left.\pi\right|_{\left(B \times f_{1} \times \cdots \times f_{m}\right)}\right)\left(X_{p}\right), d\left(\left.\pi\right|_{\left(B \times f_{1} \times \cdots \times f_{m}\right)}\right)\left(Y_{p}\right)\right),
$$

$\forall q \in B, \forall X_{p}, Y_{p} \in T_{p}\left(B \times f_{1} \times \cdots \times f_{m}\right)$, é um tensor métrico em $B \times f_{1} \times \cdots \times f_{m}$. Logo, $\left(B \times f_{1} \times \cdots \times f_{m}, g_{B \times f_{1} \times \cdots \times f_{m}}\right)$ é uma subvariedade semi-Riemanniana de 
$(M, g)$, conforme a Proposição A.16. Portanto, pela Proposição A.16, a componente de $D_{X} Y$ tangente à subvariedade $B \times f_{1} \times \cdots \times f_{m}$ é igual à conexão de Levi-Civita da subvariedade $B \times f_{1} \times \cdots \times f_{m}$ aplicada às restrições de $X$ e $Y$ a esta subvariedade. Note que $\left.\pi\right|_{\left(B \times f_{1} \times \cdots \times f_{m}\right)}:\left(B \times f_{1} \times \cdots \times f_{m}, g_{B \times f_{1} \times \cdots \times f_{m}}\right) \rightarrow\left(B, g_{B}\right)$ é uma isometria. Logo, pela Proposição A.15, $\mathrm{d} \pi\left(D_{X} Y\right)=D_{X_{B}}^{B} Y_{B}$.

(1.4) Pelo Teorema A.5, $D_{X} V=[X, V]+D_{V} X$. Pela Proposição A.1, $[X, V]=0$. Logo, $D_{X} V=D_{V} X$.

Observe que, pelo Teorema A.5,

$$
\begin{aligned}
g\left(D_{X} V, Y\right) & =X g(V, Y)-g\left(V, D_{X} Y\right) \\
& =-g\left(V, D_{X} Y\right)=0
\end{aligned}
$$

onde a última igualdade decorre de (1.3).

Além disso, se $i \neq j$, pela fórmula de Koszul, $g\left(D_{X} V, W\right)=0$. Portanto, $D_{X} V$ é tangente a $F_{i}$. Pela fórmula de Koszul,

$$
\begin{aligned}
g\left(D_{X} V, W\right) & =\frac{1}{2} X g(V, W)=\frac{1}{2} X\left[\left(b_{i} \circ \pi\right)^{2} g_{F_{i}}\left(V_{F_{i}}, W_{F_{i}}\right)\right] \\
& =\frac{1}{2}\left[X\left(b_{i} \circ \pi\right)^{2}\right] g_{F_{i}}\left(V_{F_{i}}, W_{F_{i}}\right)=\left(b_{i} \circ \pi\right)\left[X\left(b_{i} \circ \pi\right)\right] g_{F_{i}}\left(V_{F_{i}}, W_{F_{i}}\right) \\
& =\frac{X\left(b_{i} \circ \pi\right)}{\left(b_{i} \circ \pi\right)} g(V, W) .
\end{aligned}
$$

Como $W$ é arbitrário,

$$
D_{X} V=D_{V} X=\frac{X\left(b_{i} \circ \pi\right)}{\left(b_{i} \circ \pi\right)} V
$$

(1.5) Inicialmente, calcula-se a componente de $D_{V} W$ tangente à base $B$. Como $g(W, X)=0$, pelo Teorema A.5 e por (1.4),

$$
g\left(D_{V} W, X\right)=-g\left(W, D_{V} X\right)=-\frac{X\left(b_{i} \circ \pi\right)}{\left(b_{i} \circ \pi\right)} g(W, V)
$$


Mas,

$$
X\left(b_{i} \circ \pi\right)=g\left(\operatorname{grad}\left(b_{i} \circ \pi\right), X\right)
$$

Logo, como $X$ é arbitrário, tal componente é igual a $-\frac{g(V, W)}{\left(b_{i} \circ \pi\right)} \operatorname{grad}\left(b_{i} \circ \pi\right)$. Note que, se $i \neq j$, tal termo é nulo.

Para o restante da demonstração, considere dois casos, $i=j$ e $i \neq j$.

Suponha $i \neq j$. Seja $U \in \mathcal{L}\left(F_{k}\right)$. Calculando $g\left(D_{V} W, U\right)$ com o auxílio da fórmula de Koszul e da Proposição A.1, resulta que $g\left(D_{V} W, U\right)=0$ se $(i=k, j \neq k)$ ou $(j=k, i \neq k)$ ou $(i \neq j \neq k)$. Logo, como $U$ é arbitrário, $D_{V} W=0$, para $i \neq j$.

Suponha $i=j$. Seja $U \in \mathcal{L}\left(F_{k}\right)$. Calculando $g\left(D_{V} W, U\right)$ com o auxílio da fórmula de Koszul e da Proposição A.1, resulta que, se $k \neq i, j$, então $g\left(D_{V} W, U\right)=$ 0. Como $U$ é arbitrário, $D_{V} W$ é tangente a $F_{i}$.

Para calcular a componente de $D_{V} W$ tangente a $F_{i}$, fixe $i \in\{1, \cdots, m\}$. Observe que, dados $q \in B$ e $\left(f_{1}, \cdots, f_{i-1}, f_{i+1}, \cdots, f_{m}\right) \in F_{1} \times \cdots \times F_{i-1} \times F_{i+1} \times \cdots \times F_{m}$ fixos, mas, arbitrários, $q \times f_{1} \times \cdots \times f_{i-1} \times F_{i} \times f_{i+1} \times \cdots \times f_{m}$ é uma subvariedade de $M$. Considere $f_{i} \in F_{i}$ arbitrário, $p=\left(q, f_{1}, \cdots, f_{i}, \cdots, f_{m}\right)$ e a restrição da projeção canônica $\sigma_{i}: M \rightarrow F_{i}$ à subvariedade $q \times f_{1} \times \cdots \times f_{i-1} \times F_{i} \times f_{i+1} \times \cdots \times f_{m}$, isto é, $\left.\sigma_{i}\right|_{q \times f_{1} \times \cdots \times f_{i-1} \times F_{i} \times f_{i+1} \times \cdots \times f_{m}} \rightarrow F_{i}$. Então, a restrição da métrica de $M$ à subvariedade $q \times f_{1} \times \cdots \times f_{i-1} \times F_{i} \times f_{i+1} \times \cdots \times f_{m}$, dada pela aplicação

$$
g_{q \times f_{1} \times \cdots \times f_{i-1} \times F_{i} \times f_{i+1} \times \cdots \times f_{m}}:
$$

$T_{p}\left(q \times f_{1} \times \cdots \times f_{i-1} \times F_{i} \times f_{i+1} \times \cdots \times f_{m}\right) \times T_{p}\left(q \times f_{1} \times \cdots \times f_{i-1} \times F_{i} \times f_{i+1} \times \cdots \times f_{m}\right) \rightarrow \mathbb{R}$ definida por

$$
g_{q \times f_{1} \times \cdots \times f_{i-1} \times F_{i} \times f_{i+1} \times \cdots \times f_{m}}\left(V_{p}, W_{p}\right)
$$

$=b_{i}^{2}(q) g_{F_{i}}\left(d\left(\left.\sigma_{i}\right|_{\left(q \times f_{1} \times \cdots \times f_{i-1} \times F_{i} \times f_{i+1} \times \cdots \times f_{m}\right)}\right)\left(V_{p}\right), d\left(\left.\sigma_{i}\right|_{\left(q \times f_{1} \times \cdots \times f_{i-1} \times F_{i} \times f_{i+1} \times \cdots \times f_{m}\right)}\right)\left(W_{p}\right)\right)$, 
$\forall f_{i} \in F_{i}, \forall V_{p}, W_{p} \in T_{p}\left(q \times f_{1} \times \cdots \times f_{i-1} \times F_{i} \times f_{i+1} \times \cdots \times f_{m}\right)$, é um tensor métrico em $q \times f_{1} \times \cdots \times f_{i-1} \times F_{i} \times f_{i+1} \times \cdots \times f_{m}$. Logo,

$$
\left(q \times f_{1} \times \cdots \times f_{i-1} \times F_{i} \times f_{i+1} \times \cdots \times f_{m}, g_{q \times f_{1} \times \cdots \times f_{i-1} \times F_{i} \times f_{i+1} \times \cdots \times f_{m}}\right)
$$

é uma subvariedade semi-Riemanniana de $(M, g)$, conforme a Proposição A.16. Portanto, pela Proposição A.16, a componente de $D_{V} W$ tangente à subvariedade $q \times f_{1} \times \cdots \times f_{i-1} \times F_{i} \times f_{i+1} \times \cdots \times f_{m}$ é igual à conexão de Levi-Civita da subvariedade $q \times f_{1} \times \cdots \times f_{i-1} \times F_{i} \times f_{i+1} \times \cdots \times f_{m}$ aplicada às restrições de $V$ e $W$ a esta subvariedade. Note que

$$
\left.\sigma_{i}\right|_{\left(q \times f_{1} \times \cdots \times f_{i-1} \times F_{i} \times f_{i+1} \times \cdots \times f_{m}\right)}:
$$

$$
\left(q \times f_{1} \times \cdots \times f_{i-1} \times F_{i} \times f_{i+1} \times \cdots \times f_{m}, g_{q \times f_{1} \times \cdots \times f_{i-1} \times F_{i} \times f_{i+1} \times \cdots \times f_{m}}\right) \rightarrow\left(F_{i}, g_{F_{i}}\right)
$$

é uma homotetia, pois, neste caso, o ponto $q$ está fixo e, portanto, $b_{i}(q)$ é uma constante. Logo, pela Proposição A.15, a componente de $D_{V} W$ tangente a $F_{i}$ é igual a $\overline{D_{V_{F_{i}}}^{F_{i}} W_{F_{i}}}$.

Proposição 1.4 Dados $M=B \times{ }_{b_{1}} F_{1} \times \cdots \times{ }_{b_{m}} F_{m}, \phi \in \mathcal{F}(B)$ e $X, Y \in \mathcal{L}(B)$, então

$$
H^{\phi \circ \pi}(X, Y)=H_{B}^{\phi}\left(X_{B}, Y_{B}\right) \circ \pi=\overline{H_{B}^{\phi}\left(X_{B}, Y_{B}\right)}
$$

\section{Demonstração:}

$$
\begin{aligned}
H^{\phi \circ \pi}(X, Y) & =g\left(D_{X} \operatorname{grad}(\phi \circ \pi), Y\right)=g\left(\overline{D_{X_{B}}^{B} \operatorname{grad}_{B} \phi}, Y\right) \\
& =g_{B}\left(D_{X_{B}}^{B} \operatorname{grad}_{B} \phi, Y_{B}\right) \circ \pi=H_{B}^{\phi}\left(X_{B}, Y_{B}\right) \circ \pi .
\end{aligned}
$$


Proposição 1.5 Dados $M=B \times{ }_{b_{1}} F_{1} \times \cdots \times{ }_{b_{m}} F_{m}, X, Y, Z \in \mathcal{L}(B), V \in \mathcal{L}\left(F_{i}\right)$, $W \in \mathcal{L}\left(F_{j}\right)$ e $U \in \mathcal{L}\left(F_{k}\right)$, então, $\forall i, j, k \in\{1, \ldots, m\}$,

$$
\begin{aligned}
& R(V, X) Y=-R(X, V) Y=-\frac{H^{b_{i} \circ \pi}(X, Y)}{\left(b_{i} \circ \pi\right)} V \\
& R(X, Y) Z=\overline{R^{B}\left(X_{B}, Y_{B}\right) Z_{B}}, \\
& R(V, W) X=0 \text {, } \\
& R(X, Y) V=0 \\
& R(X, V) W=R(V, X) W=0, \text { se } i \neq j, \\
& R(X, V) W=-R(V, X) W=-\frac{g(V, W)}{\left(b_{i} \circ \pi\right)} \overline{D_{X_{B}}^{B} \operatorname{grad}_{B} b_{i}}, \text { se } i=j, \\
& R(V, W) U=0, \text { se } i \neq j, i \neq k \text { e } j \neq k, \\
& R(V, W) U=0, \text { se } i=j \text { e } i, j \neq k, \\
& R(U, V) W=-R(V, U) W=-g(V, W) \frac{\left[g_{B}\left(\operatorname{grad}_{B} b_{i}, \operatorname{grad}_{B} b_{k}\right) \circ \pi\right]}{\left(b_{i} b_{k} \circ \pi\right)} U \\
& \text { se } i=j e i, j \neq k \quad e \\
& R(V, W) U=\overline{R^{F_{i}}\left(V_{F_{i}}, W_{F_{i}}\right) U_{F_{i}}}+ \\
& \frac{\left[g_{B}\left(\operatorname{grad}_{B} b_{i}, \operatorname{grad}_{B} b_{i}\right) \circ \pi\right]}{\left(b_{i} \circ \pi\right)^{2}}[g(V, U) W-g(W, U) V], \\
& \text { se } i=j=k \text {. }
\end{aligned}
$$

\section{Demonstração:}

(1.6) Pela Proposição A.1, $[V, X]=0$. Portanto, $R(V, X) Y=D_{V} D_{X} Y-D_{X} D_{V} Y$.

Utilizando a Proposição 1.3,

$$
\begin{aligned}
D_{X} D_{V} Y & =D_{X}\left[\frac{Y\left(b_{i} \circ \pi\right)}{b_{i} \circ \pi} V\right] \\
& =\left[\frac{X Y\left(b_{i} \circ \pi\right)}{\left(b_{i} \circ \pi\right)}-\frac{X\left(b_{i} \circ \pi\right) Y\left(b_{i} \circ \pi\right)}{\left(b_{i} \circ \pi\right)^{2}}+\frac{Y\left(b_{i} \circ \pi\right)}{\left(b_{i} \circ \pi\right)} \frac{X\left(b_{i} \circ \pi\right)}{\left(b_{i} \circ \pi\right)}\right] V \\
& =\frac{X Y\left(b_{i} \circ \pi\right)}{\left(b_{i} \circ \pi\right)} V
\end{aligned}
$$

e

$$
D_{V} D_{X} Y=\frac{D_{X} Y\left(b_{i} \circ \pi\right)}{\left(b_{i} \circ \pi\right)} V
$$


Logo, pela Proposição A.13,

$$
R(V, X) Y=-\frac{H^{b_{i} \circ \pi}(X, Y)}{\left(b_{i} \circ \pi\right)} V
$$

(1.7) Utilizando (1.6) e a Proposição A.7,

$$
g(R(X, Y) Z, V)=g(R(Z, V) X, Y)=0
$$

Como $V$ é arbitrário, $R(X, Y) Z$ é tangente à base $B$.

Dados $f_{i} \in F_{i}, \forall i \in\{1, \ldots, m\}$ fixos, mas, arbitrários, $B \times f_{1} \times \cdots \times f_{m}$ é uma subvariedade semi-Riemanniana de $M$, conforme apresentado na demonstração da equação (1.3) da Proposição 1.3. Como $D_{X} Y$ é tangente à base $B$, segue que, conforme a Proposição A.17, $\Pi(X, Y)=0, \forall X, Y \in \mathcal{L}(B)$. Portanto, $R(X, Y) Z$ coincide com a aplicação do tensor de Riemann da subvariedade $B \times f_{1} \times \cdots \times f_{m}$ às restrições dos campos $X, Y$ e $Z$ a esta subvariedade, como uma conseqüiência da equação de Gauss.

Mas, a restrição da projeção canônica $\pi: M \rightarrow B$ à subvariedade $B \times f_{1} \times \cdots \times f_{m}$, isto é, $\left.\pi\right|_{B \times f_{1} \times \cdots \times f_{m}} \rightarrow B$, é uma isometria e, conforme a Proposição A.15, o tensor de Riemann é preservado. Logo, $R(X, Y) Z=\overline{R^{B}\left(X_{B}, Y_{B}\right) Z_{B}}$.

(1.8) Existem dois casos distintos, $i \neq j$ e $i=j$. Suponha $i \neq j$. Então, conforme a Proposição A.1,

$$
\begin{aligned}
R(V, W) X & =D_{V} D_{W} X-D_{W} D_{V} X \\
& =V\left(\frac{X\left(b_{j} \circ \pi\right)}{\left(b_{j} \circ \pi\right)}\right) W-W\left(\frac{X\left(b_{i} \circ \pi\right)}{\left(b_{i} \circ \pi\right)}\right) V=0,
\end{aligned}
$$

pois $\left(\frac{X\left(b_{i} \circ \pi\right)}{\left(b_{i} \circ \pi\right)}\right)$ é constante nas fibras, $\forall i \in\{1, \ldots, m\}$.

Suponha $i=j$. Neste caso, conforme mencionado nas Proposições 3.36 e 7.42 do livro de O'Neill [22], é possível considerar $[V, W]=0$ no cálculo. Isto ocorre, pois, 
como as identidades tensoriais têm caráter local, no sentido descrito na Proposição A.18, dado um ponto $p \in M$ e vetores $v, w \in T_{p} M$, é possível estender convenientemente estes vetores em uma certa vizinhança a campos $V, W$ com componentes constantes em um dado sistema de coordenadas. Segue que $[V, W]=0$. Neste caso, $R(V, W) X=V\left(\frac{X\left(b_{i} \circ \pi\right)}{\left(b_{i} \circ \pi\right)}\right) W-W\left(\frac{X\left(b_{i} \circ \pi\right)}{\left(b_{i} \circ \pi\right)}\right) V+\frac{X\left(b_{i} \circ \pi\right)}{\left(b_{i} \circ \pi\right)}\left(D_{V} W-D_{W} V\right)=0$ pois, conforme o Teorema A.5, $D_{V} W-D_{W} V=[V, W]=0$.

(1.9) Utilizando o resultado (1.8),

$$
g(R(X, Y) V, W)=g(R(V, W) X, Y)=0
$$

Utilizando o resultado (1.7),

$$
g(R(X, Y) V, Z)=-g(R(X, Y) Z, V)=0
$$

Logo, como $W$ e $Z$ são arbitrários,

$$
R(X, Y) V=0 .
$$

(1.10) Utilizando o resultado (1.8),

$$
g(R(X, V) W, U)=g(R(W, U) X, V)=0 .
$$

Utilizando o resultado (1.6),

$$
\begin{aligned}
g(R(X, V) W, Y) & =-g(R(X, V) Y, W) \\
& =-\frac{H^{b_{i} \circ \pi}(X, Y)}{\left(b_{i} \circ \pi\right)} g(V, W)=0,
\end{aligned}
$$

pois $g(V, W)=0$. 
(1.11) Conforme a discussão em (1.10),

$$
\begin{aligned}
g(R(X, V) W, U) & =0 \mathrm{e} \\
g(R(X, V) W, Y) & =-\frac{H^{b_{i} \circ \pi}(X, Y)}{\left(b_{i} \circ \pi\right)} g(V, W) \\
& =-\frac{g\left(D_{X} \operatorname{grad}\left(b_{i} \circ \pi\right), Y\right)}{\left(b_{i} \circ \pi\right)} g(V, W) .
\end{aligned}
$$

Como $Y$ é arbitrário, utilizando a equação (1.3) da Proposição 1.3, segue o resultado.

(1.12) O resultado segue do fato de que $D_{V} W=0, \forall V \in \mathcal{L}\left(F_{i}\right), W \in \mathcal{L}\left(F_{j}\right)$ se $i \neq j$ e do fato de que $[V, W]=0$ nas mesmas condições, conforme a Proposição A.1.

(1.13) Conforme a Proposição A.1, $[V, W] \in \mathcal{L}\left(F_{i}\right)$. Logo, $D_{[V, W]} U=0$. Além disso, $D_{W} U=D_{V} U=0$, o que implica no resultado.

(1.14) $R(U, V) W=D_{U} D_{V} W$, pois $[U, V]=0$ e $D_{U} W=0$. Portanto,

$$
R(U, V) W=D_{U}\left(-\frac{g(V, W)}{\left(b_{i} \circ \pi\right)} \operatorname{grad}\left(b_{i} \circ \pi\right)\right)
$$

pois $D_{U}\left(\overline{D_{V_{F_{i}}}^{F_{i}} W_{F_{i}}}\right)=0$. Logo,

$$
R(U, V) W=-\frac{g(V, W)}{\left(b_{i} \circ \pi\right)} \frac{\left[\operatorname{grad}\left(b_{i} \circ \pi\right)\right]\left(b_{k} \circ \pi\right)}{\left(b_{k} \circ \pi\right)} U
$$

pois a função $\frac{g(V, W)}{\left(b_{i} \circ \pi\right)}$ é constante na fibra $F_{k}$.

O resultado segue do fato de que

$$
\left[\operatorname{grad}\left(b_{i} \circ \pi\right)\right]\left(b_{k} \circ \pi\right)=\left[g_{B}\left(\operatorname{grad}_{B} b_{i}, \operatorname{grad}_{B} b_{k}\right) \circ \pi\right]
$$

(1.15) Utilizando (1.8),

$$
g(R(V, W) U, X)=-g(R(V, W) X, U)=0 .
$$


Seja $T \in \mathcal{L}\left(F_{l}\right)$, onde $l \neq i, j, k$. Então,

$$
g(R(V, W) U, T)=-g(R(V, W) T, U)=0
$$

como conseqüência de (1.13).

Logo, $R(V, W) U$ é tangente a $F_{i}$, pois $X$ e $T$ são arbitrários.

Dados $q \in B$ e $\left(f_{1}, \cdots, f_{i-1}, f_{i+1}, \cdots, f_{m}\right) \in F_{1} \times \cdots \times F_{i-1} \times F_{i+1} \times \cdots \times F_{m}$ fixos, mas, arbitrários, $q \times f_{1} \times \cdots \times f_{i-1} \times F_{i} \times f_{i+1} \times \cdots \times f_{m}$ é uma subvariedade semiRiemanniana de $M$, conforme apresentado na demonstração da equação (1.5) da Proposição 1.3. Além disso, a restrição da projeção canônica $\sigma_{i}: M \rightarrow F_{i}$ à subvariedade $q \times f_{1} \times \cdots \times f_{i-1} \times F_{i} \times f_{i+1} \times \cdots \times f_{m}$, isto é, $\left.\sigma_{i}\right|_{q \times f_{1} \times \cdots \times f_{i-1} \times F_{i} \times f_{i+1} \times \cdots \times f_{m}} \rightarrow F_{i}$, é uma homotetia, conforme discutido no mesmo local. Portanto, $R(V, W) U$ e $\overline{R_{F_{i}}\left(V_{F_{i}}, W_{F_{i}}\right) U_{F_{i}}}$ estão relacionados pela equação de Gauss, conforme as Proposições A.15 e A.17, onde

$$
\Pi(V, W)=-\frac{g(V, W)}{\left(b_{i} \circ \pi\right)} \operatorname{grad}\left(b_{i} \circ \pi\right)
$$

Dado $T \in \mathcal{L}\left(F_{i}\right)$ arbitrário,

$$
\begin{aligned}
g(R(V, W) U, T)= & g\left(\overline{R_{F_{i}}\left(V_{F_{i}}, W_{F_{i}}\right) U_{F_{i}}}, T\right) \\
& +g\left(-\frac{g(V, U)}{\left(b_{i} \circ \pi\right)} \operatorname{grad}\left(b_{i} \circ \pi\right),-\frac{g(W, T)}{\left(b_{i} \circ \pi\right)} \operatorname{grad}\left(b_{i} \circ \pi\right)\right) \\
& -g\left(-\frac{g(V, T)}{\left(b_{i} \circ \pi\right)} \operatorname{grad}\left(b_{i} \circ \pi\right),-\frac{g(W, U)}{\left(b_{i} \circ \pi\right)} \operatorname{grad}\left(b_{i} \circ \pi\right)\right),
\end{aligned}
$$

o que leva à identidade desejada.

Observação: O resultado em (1.11) na Proposição 1.5 difere por um sinal do apresentado no artigo de Dobarro e Ünal [13].

Proposição 1.6 Dados $M=B \times{ }_{b_{1}} F_{1} \times \cdots \times{ }_{b_{m}} F_{m}, \phi \in \mathcal{F}(B)$ e $\psi_{i} \in \mathcal{F}\left(F_{i}\right)$, 
então, $\forall i \in\{1, \ldots, m\}$,

$$
\begin{aligned}
\Delta(\phi \circ \pi) & =\left(\Delta_{B} \phi\right) \circ \pi+\sum_{i=1}^{m} s_{i} \frac{g_{B}\left(\operatorname{grad}_{B} \phi, \operatorname{grad}_{B} b_{i}\right)}{b_{i}} \circ \pi \quad e \\
\Delta\left(\psi_{i} \circ \sigma_{i}\right) & =\frac{\left(\Delta_{F_{i}} \psi_{i}\right) \circ \sigma_{i}}{\left(b_{i} \circ \pi\right)^{2}}
\end{aligned}
$$

onde $s_{i}=\operatorname{dim}\left(F_{i}\right)$.

Demonstração: Pela definição de Laplaciano de uma função e pela Proposição A.12, tem-se

(1.16) $\Delta(\phi \circ \pi)=\sum_{j=1}^{n} g\left(E_{j}, E_{j}\right) g\left(D_{E_{j}} \overline{\operatorname{grad}_{B} \phi}, E_{j}\right)$, onde $n=\operatorname{dim}(M)$ e $\left\{E_{j}\right\}$ é um referencial ortonormal móvel, conforme a Proposição A.3.

A soma pode ser dividida em partes. Em primeiro lugar, considere valores de $j$ tais que $E_{j}$ é tangente à base $B$. Neste caso, a contribuição para a soma é dada por

$$
\sum g_{B}\left(\mathrm{~d} \pi\left(E_{j}\right), \mathrm{d} \pi\left(E_{j}\right)\right) g_{B}\left(D_{\mathrm{d} \pi\left(E_{j}\right)}^{B} \operatorname{grad}_{B} \phi, \mathrm{d} \pi\left(E_{j}\right)\right) \circ \pi=\left(\Delta_{B} \phi\right) \circ \pi .
$$

Considere valores de $j$ tais que $E_{j}$ é tangente à fibra $F_{i}$, com $i$ fixo. A contribuição, neste caso, é dada por

$$
\sum g\left(E_{j}, E_{j}\right) g\left(\frac{\overline{\operatorname{grad}_{B} \phi}\left(b_{i} \circ \pi\right)}{\left(b_{i} \circ \pi\right)} E_{j}, E_{j}\right)=s_{i} \frac{g_{B}\left(\operatorname{grad}_{B} \phi, \operatorname{grad}_{B} b_{i}\right)}{b_{i}} \circ \pi,
$$

pois $g\left(E_{j}, E_{j}\right)= \pm 1$, logo, $g^{2}\left(E_{j}, E_{j}\right)=1$.

Somando sobre todos os valores de $i$, resulta na fórmula desejada.

(1.17) Usando a definição de Laplaciano, a Definição A.12 e a Proposição 1.2, equação (1.2), obtém-se

$$
\begin{aligned}
\Delta\left(\psi_{i} \circ \sigma_{i}\right)= & \sum_{j=1}^{n} g\left(E_{j}, E_{j}\right) g\left(D_{E_{j}}\left(\overline{\frac{\operatorname{grad}_{F_{i}} \psi_{i}}{\left(b_{i} \circ \pi\right)^{2}}}\right), E_{j}\right) \\
= & \sum_{j=1}^{n}\left\{g\left(E_{j}, E_{j}\right) E_{j}\left(\frac{1}{b_{i} \circ \pi}\right)^{2} g\left(\overline{\operatorname{grad}_{F_{i}} \psi_{i}}, E_{j}\right)\right. \\
& \left.+\frac{g\left(E_{j}, E_{j}\right)}{\left(b_{i} \circ \pi\right)^{2}} g\left(D_{E_{j}} \overline{\operatorname{grad}_{F_{i}} \psi_{i}}, E_{j}\right)\right\} .
\end{aligned}
$$


Note que, para valores de $j$ tais que $E_{j}$ é tangente à base $B$, a contribuição é nula, conforme a Proposição 1.3 e a expressão da métrica. Considere valores de $j$ tais que $E_{j}$ é tangente à fibra $F_{k}$, com $k$ fixo. Neste caso, $E_{j}\left(\frac{1}{b_{i} \circ \pi}\right)^{2}=0$, pois $\left(\frac{1}{b_{i} \circ \pi}\right)^{2}$ é constante na fibra $F_{k}$. Conforme (1.5) da Proposição 1.3, a única contribuição não nula é obtida de valores de $j$ tais que $E_{j}$ é tangente à fibra $F_{i}$. Neste caso, a contribuição é

$$
\begin{aligned}
& \frac{1}{\left(b_{i} \circ \pi\right)^{2}} \sum g\left(E_{j}, E_{j}\right) g\left(D_{E_{j}} \overline{\operatorname{grad}_{F_{i}} \psi_{i}}, E_{j}\right) \\
& =\frac{1}{\left(b_{i} \circ \pi\right)^{2}} \sum\left(b_{i} \circ \pi\right)^{2} g_{F_{i}}\left(\mathrm{~d} \sigma_{i}\left(E_{j}\right), \mathrm{d} \sigma_{i}\left(E_{j}\right)\right)\left(b_{i} \circ \pi\right)^{2} g_{F_{i}}\left(D_{\mathrm{d} \sigma_{i}\left(E_{j}\right)}^{F_{i}} \operatorname{grad}_{F_{i}} \psi_{i}, \mathrm{~d} \sigma_{i}\left(E_{j}\right)\right) .
\end{aligned}
$$

Note que, para valores de $j$ tais que $E_{j}$ é tangente à fibra $F_{i},\left\{b_{i} \mathrm{~d} \sigma_{i}\left(E_{j}\right)\right\}$ forma uma base ortonormal do espaço tangente à fibra $F_{i}$, pois

$$
g_{F_{i}}\left(b_{i} \mathrm{~d} \sigma_{i}\left(E_{j}\right), b_{i} \mathrm{~d} \sigma_{i}\left(E_{j}\right)\right)=b_{i}^{2} \frac{g\left(E_{j}, E_{j}\right)}{b_{i}^{2}}= \pm 1
$$

Portanto,

$$
\Delta\left(\psi_{i} \circ \sigma_{i}\right)=\frac{\left(\Delta_{F_{i}} \psi_{i}\right) \circ \sigma_{i}}{\left(b_{i} \circ \pi\right)^{2}}
$$

Proposição 1.7 Dados $M=B \times{ }_{b_{1}} F_{1} \times \cdots \times{ }_{b_{m}} F_{m}, X, Y, Z \in \mathcal{L}(B), V \in \mathcal{L}\left(F_{i}\right)$ e $W \in \mathcal{L}\left(F_{j}\right)$, então, $\forall i, j \in\{1, \ldots, m\}$,

$$
\begin{aligned}
\operatorname{Ric}(X, Y)= & \overline{\operatorname{Ric}^{B}\left(X_{B}, Y_{B}\right)}-\sum_{i=1}^{m} \frac{s_{i}}{\left(b_{i} \circ \pi\right)} H^{b_{i} \circ \pi}(X, Y) \\
\operatorname{Ric}(X, V)= & \operatorname{Ric}(V, X)=0 \\
\operatorname{Ric}(V, W)= & 0, \text { se } i \neq j e \\
\operatorname{Ric}(V, W)= & \overline{\operatorname{Ric}^{F_{i}}\left(V_{F_{i}}, W_{F_{i}}\right)} \\
& -g(V, W)\left\{\frac{\left(\Delta_{B} b_{i}\right) \circ \pi}{\left(b_{i} \circ \pi\right)}+\left(s_{i}-1\right)\left[\frac{g_{B}\left(\operatorname{grad}_{B} b_{i}, \operatorname{grad}_{B} b_{i}\right)}{b_{i}^{2}}\right] \circ \pi\right. \\
& \left.+\sum_{k=1, k \neq i}^{m} s_{k}\left[\frac{g_{B}\left(\operatorname{grad}_{B} b_{i}, \operatorname{grad}_{B} b_{k}\right)}{b_{i} b_{k}}\right] \circ \pi\right\}, \text { se } i=j
\end{aligned}
$$

onde $s_{i}=\operatorname{dim}\left(F_{i}\right)$. 
Demonstração: (1.18) $\operatorname{Ric}(X, Y)=-\sum_{k=1}^{n} g\left(E_{k}, E_{k}\right) g\left(R\left(X, E_{k}\right) Y, E_{k}\right)$, conforme a Proposição A.8, onde $n=\operatorname{dim}(M)$ e $\left\{E_{k}\right\}$ é um referencial ortonormal móvel.

Considere os valores de $k$ tais que $E_{k}$ é tangente à base $B$. Neste caso, conforme a Proposição 1.5, equação (1.7), a contribuição total é $\overline{\operatorname{Ric}^{B}\left(X_{B}, Y_{B}\right)}$.

Considere valores de $k$ tais que $E_{k}$ é tangente à fibra $i$, com $i$ fixo. Neste caso, a contribuição é, conforme a Proposição 1.5, equação (1.6),

$$
-\sum g\left(E_{k}, E_{k}\right) \frac{H^{b_{i} \circ \pi}(X, Y)}{\left(b_{i} \circ \pi\right)} g\left(E_{k}, E_{k}\right)=-\frac{s_{i}}{\left(b_{i} \circ \pi\right)} H^{b_{i} \circ \pi}(X, Y) .
$$

Somando sobre todos os valores de $i$ resulta na expressão desejada.

(1.19) Conforme a Proposição 1.5, equação (1.8), a contribuição para $\operatorname{Ric}(V, X)$ de valores de $k$ tais que $E_{k}$ é tangente a qualquer uma das fibras é nula.

Considere valores de $k$ tais que $E_{k}$ é tangente à base $B$. Neste caso, conforme a Proposição 1.5, equação (1.6), $R\left(V, E_{k}\right) X$ é tangente à fibra $F_{i}$. Logo, $g\left(R\left(V, E_{k}\right) X, E_{k}\right)$ é nulo. Portanto, $\operatorname{Ric}(V, X)=\operatorname{Ric}(X, V)=0$.

(1.20) Considere valores de $k$ tais que $E_{k}$ é tangente à base $B$. Neste caso, a contribuição é nula, conforme a Proposição 1.5, equação (1.10).

Considere valores de $k$ tais que $E_{k}$ é tangente à fibra $F_{s}$, com $s \in\{1, \ldots, m\}$. Neste caso, conforme a Proposição 1.5 , equações (1.12), (1.13) e (1.14), $R\left(V, E_{k}\right) W$ é não nulo somente se $E_{k}$ é tangente à fibra $F_{j}$ e, então, $R\left(V, E_{k}\right) W$ é tangente à fibra $F_{i}$. Mas, neste caso, $g\left(R\left(V, E_{k}\right) W, E_{k}\right)$ é nulo. Portanto, esta contribuição é, também, nula.

(1.21) Considere valores de $k$ tais que $E_{k}$ é tangente à base $B$. Neste caso, conforme 
a Proposição 1.5, equação (1.11), a contribuição é

$$
-\sum g\left(E_{k}, E_{k}\right) \frac{g(V, W)}{\left(b_{i} \circ \pi\right)} g\left(\overline{D_{\mathrm{d} \pi\left(E_{k}\right)}^{B} \operatorname{grad}_{B} b_{i}}, E_{k}\right)=-\frac{g(V, W)}{\left(b_{i} \circ \pi\right)}\left[\left(\Delta_{B} b_{i}\right) \circ \pi\right]
$$

Considere valores de $k$ tais que $E_{k}$ é tangente à fibra $F_{i}$. Neste caso, conforme a Proposição 1.5, equação (1.15), a contribuição é

$$
\begin{aligned}
& -\sum g\left(E_{k}, E_{k}\right) g\left(\overline{R^{F_{i}}\left(V_{F_{i}}, \mathrm{~d} \sigma_{i}\left(E_{k}\right)\right) W_{F_{i}}}, E_{k}\right) \\
& -\sum g\left(E_{k}, E_{k}\right) \frac{\left[g_{B}\left(\operatorname{grad}_{B} b_{i}, \operatorname{grad}_{B} b_{i}\right) \circ \pi\right]}{\left(b_{i} \circ \pi\right)^{2}} g\left(g(V, W) E_{k}-g\left(E_{k}, W\right) V, E_{k}\right) .
\end{aligned}
$$

Note que na expressão acima, a parte da última parcela dada por

$$
\sum g\left(E_{k}, E_{k}\right) g\left(E_{k}, W\right) g\left(V, E_{k}\right)
$$

com $k$ restrito a valores tais que $E_{k}$ é tangente à fibra $i$ é igual à mesma soma com $k$ tomando todos os valores possíveis de 1 a $\operatorname{dim}(M)$. Isto ocorre pois $g\left(E_{k}, W\right)$ e $g\left(V, E_{k}\right)$ são nulos se $E_{k}$ não é tangente à fibra $F_{i}$. Logo, conforme a Proposição A.3,

$$
\sum g\left(E_{k}, E_{k}\right) g\left(E_{k}, W\right) g\left(V, E_{k}\right)=g(V, W)
$$

Portanto, estes valores de $k$ contribuem com

$$
\overline{\operatorname{Ric}^{F_{i}}\left(V_{F_{i}}, W_{F_{i}}\right)}-g(V, W)\left(s_{i}-1\right) \frac{\left[g_{B}\left(\operatorname{grad}_{B} b_{i}, \operatorname{grad}_{B} b_{i}\right) \circ \pi\right]}{\left(b_{i} \circ \pi\right)^{2}} .
$$

Considere valores de $k$ tais que $E_{k}$ é tangente à fibra $F_{r} \operatorname{com} r \in\{1, \ldots, m\}$, $r \neq i$. Logo, conforme a Proposição 1.5, equação (1.14), a contribuição é, para $r$ fixo,

$$
\begin{aligned}
& -\sum g\left(E_{k}, E_{k}\right) \frac{g(V, W)}{\left(b_{i} b_{r} \circ \pi\right)}\left[g_{B}\left(\operatorname{grad}_{B} b_{i}, \operatorname{grad}_{B} b_{r}\right) \circ \pi\right] g\left(E_{k}, E_{k}\right) \\
& =-\frac{g(V, W)}{\left(b_{i} b_{r} \circ \pi\right)}\left[g_{B}\left(\operatorname{grad}_{B} b_{i}, \operatorname{grad}_{B} b_{r}\right) \circ \pi\right] s_{r} .
\end{aligned}
$$


Somando sobre todos os valores de $r$, com $r \neq i$, resulta

$$
-g(V, W)\left(\sum_{r=1, r \neq i}^{m} s_{r}\left[\frac{g_{B}\left(\operatorname{grad}_{B} b_{i}, \operatorname{grad}_{B} b_{r}\right)}{b_{i} b_{r}}\right] \circ \pi\right) .
$$

A soma de todos os termos resulta na expressão desejada.

Proposição 1.8 Seja $M=B \times{ }_{b_{1}} F_{1} \times \cdots \times{ }_{b_{m}} F_{m}$. Então, a curvatura escalar $S$ admite as seguintes expressões:

$$
\begin{aligned}
S= & \left(S_{B} \circ \pi\right)-2 \sum_{i=1}^{m} s_{i} \frac{\left(\Delta_{B} b_{i}\right) \circ \pi}{\left(b_{i} \circ \pi\right)}+\sum_{i=1}^{m} \frac{\left(S_{F_{i}} \circ \sigma_{i}\right)}{\left(b_{i} \circ \pi\right)^{2}} \\
& -\sum_{i=1}^{m} \frac{s_{i}\left(s_{i}-1\right)}{\left(b_{i} \circ \pi\right)^{2}}\left[g_{B}\left(\operatorname{grad}_{B} b_{i}, \operatorname{grad}_{B} b_{i}\right) \circ \pi\right] \\
& -\sum_{i=1}^{m} \sum_{k=1, k \neq i}^{m} s_{k} s_{i} \frac{\left[g_{B}\left(\operatorname{grad}_{B} b_{i}, \operatorname{grad}_{B} b_{k}\right) \circ \pi\right]}{\left(b_{i} b_{k} \circ \pi\right)} e \\
S= & \left(S_{B} \circ \pi\right)-\sum_{i=1}^{m} \frac{\left(\Delta_{B} b_{i}\right) \circ \pi}{\left(b_{i} \circ \pi\right)}+\sum_{i=1}^{m} \frac{\left(S_{F_{i}} \circ \sigma_{i}\right)}{\left(b_{i} \circ \pi\right)^{2}} \\
& -\left[\operatorname{div}_{B}\left(\sum_{i=1}^{m} \frac{s_{i} \operatorname{grad}_{B} b_{i}}{b_{i}}\right)\right] \circ \pi \\
& -\left[g_{B}\left(\sum_{i=1}^{m} \frac{s_{i} \operatorname{grad}_{B} b_{i}}{b_{i}}, \sum_{i=1}^{m} \frac{\left.s_{i} \operatorname{grad}_{B} b_{i}\right)}{b_{i}}\right)\right] \circ \pi,
\end{aligned}
$$

onde $s_{i}=\operatorname{dim}\left(F_{i}\right)$.

Demonstração: (1.22) Sejam $\left\{E_{1}^{B}, \ldots, E_{s}^{B}\right\}$ e $\left\{E_{1}^{F_{i}}, \ldots, E_{s_{i}}^{F_{i}}\right\}$ referenciais ortonormais móveis em conjuntos abertos $U \subseteq B$ e $V_{i} \subseteq F_{i}, \forall i \in\{1, \cdots, m\}$, respectivamente, onde $s=\operatorname{dim}(B)$. Então,

$$
\left\{\overline{E_{1}^{B}}, \ldots, \overline{E_{s}^{B}}, \frac{1}{b_{1} \circ \pi} \overline{E_{1}^{F_{1}}}, \ldots, \frac{1}{b_{1} \circ \pi} \overline{E_{s_{1}}^{F_{1}}}, \ldots, \frac{1}{b_{m} \circ \pi} \overline{E_{1}^{F_{m}}}, \ldots, \frac{1}{b_{m} \circ \pi} \overline{E_{s_{m}}^{F_{m}}}\right\}
$$

é um referencial ortonormal móvel em um conjunto aberto $W \subseteq U \times V_{1} \times \cdots \times V_{m} \subseteq$ M. Conforme a Proposição A.9,

$$
S=\sum_{i=1}^{n} g\left(E_{i}, E_{i}\right) \operatorname{Ric}\left(E_{i}, E_{i}\right)
$$


onde $\left\{E_{i}\right\}$ é um referencial ortonormal móvel e $n=\operatorname{dim}(M)$.

Utilizando a Proposição 1.7, equações (1.18) e (1.21) e o referencial ortonormal móvel acima definido,

$$
\begin{aligned}
& S=\left[\sum_{i=1}^{s} g_{B}\left(E_{i}^{B}, E_{i}^{B}\right)\left[\operatorname{Ric}^{B}\left(E_{i}^{B}, E_{i}^{B}\right)-\sum_{j=1}^{m} \frac{s_{j}}{b_{j}} H_{B}^{b_{j}}\left(E_{i}^{B}, E_{i}^{B}\right)\right]\right] \circ \pi \\
& +\sum_{i=1}^{m} \sum_{j=1}^{s_{i}}\left[g_{F_{i}}\left(E_{j}^{F_{i}}, E_{j}^{F_{i}}\right) \operatorname{Ric}^{F_{i}}\left(\frac{1}{b_{i}} E_{j}^{F_{i}}, \frac{1}{b_{i}} E_{j}^{F_{i}}\right)\right] \\
& -\sum_{i=1}^{m} \sum_{j=1}^{s_{i}}\left(g_{F_{i}}\left(E_{j}^{F_{i}}, E_{j}^{F_{i}}\right)\right)^{2}\left(\frac{\left(\Delta_{B} b_{i}\right) \circ \pi}{\left(b_{i} \circ \pi\right)}+\left(s_{i}-1\right)\left[\frac{g_{B}\left(\operatorname{grad}_{B} b_{i}, \operatorname{grad}_{B} b_{i}\right)}{b_{i}^{2}}\right] \circ \pi\right. \\
& \left.+\sum_{k=1, k \neq i}^{m} s_{k}\left[\frac{g_{B}\left(\operatorname{grad}_{B} b_{i}, \operatorname{grad}_{B} b_{k}\right)}{b_{i} b_{k}}\right] \circ \pi\right)
\end{aligned}
$$

onde

$$
g\left(\frac{1}{b_{i}} \overline{E_{j}^{F_{i}}}, \frac{1}{b_{i}} \overline{E_{j}^{F_{i}}}\right)=g_{F_{i}}\left(E_{j}^{F_{i}}, E_{j}^{F_{i}}\right) .
$$

Notando que $\operatorname{Ric}^{F_{i}}$ é um tensor, portanto, linear,

$$
\left(g_{F_{i}}\left(E_{j}^{F_{i}}, E_{j}^{F_{i}}\right)\right)^{2}=1, g_{B}\left(E_{i}^{B}, E_{i}^{B}\right)=\left(g_{B}\right)_{i i}=\left(g_{B}\right)^{i i}
$$

onde $\left(g_{B}\right)^{i j}$ é a matriz inversa de $\left(g_{B}\right)_{i j}$, pois $\left(g_{B}\right)_{i i}= \pm 1 \mathrm{e}$

$$
\sum_{i=1}^{s} g_{B}\left(E_{i}^{B}, E_{i}^{B}\right) H_{B}^{b_{j}}\left(E_{i}^{B}, E_{i}^{B}\right)=\Delta_{B} b_{j}
$$

resulta na equação (1.22).

(1.23) A segunda equação resulta do fato que

$$
\operatorname{div}_{B}\left(\sum_{i=1}^{m} \frac{s_{i}}{b_{i}} \operatorname{grad}_{B} b_{i}\right)=\sum_{i=1}^{m} \frac{s_{i}}{b_{i}} \Delta_{B} b_{i}-\sum_{i=1}^{m} \frac{s_{i}}{b_{i}^{2}} g_{B}\left(\operatorname{grad}_{B} b_{i}, \operatorname{grad}_{B} b_{i}\right),
$$

pois, conforme a Proposição A.12 e a Proposição A.3,

$$
\begin{aligned}
& \sum_{j=1}^{s} g_{B}\left(E_{j}^{B}, E_{j}^{B}\right) E_{j}^{B}\left(\frac{s_{i}}{b_{i}}\right) g_{B}\left(\operatorname{grad}_{B} b_{i}, E_{j}^{B}\right) \\
& =-\sum_{j=1}^{s} g_{B}\left(E_{j}^{B}, E_{j}^{B}\right) \frac{s_{i}}{b_{i}^{2}}\left(g_{B}\left(\operatorname{grad}_{B} b_{i}, E_{j}^{B}\right)\right)^{2}=-\frac{s_{i}}{b_{i}^{2}} g_{B}\left(\operatorname{grad}_{B} b_{i}, \operatorname{grad}_{B} b_{i}\right) .
\end{aligned}
$$




\section{Capítulo 2}

\section{Espaços-tempo de Robertson-Walker generalizados}

Os principais resultados deste capítulo estão contidos na referência [13]. Considere a seguinte definição:

Definição 2.1 Considere um produto multiplamente torcido $M=B \times{ }_{b_{1}} F_{1} \times \cdots \times$ ${ }_{b_{m}} F_{m}$. Se $B$ for um intervalo aberto conexo da forma $I=\left(t_{1}, t_{2}\right)$, munido da métrica $g_{B}=-d t \otimes d t, \forall t \in I,-\infty \leq t_{1}<t_{2} \leq \infty$ e $\left(F_{i}, g_{F_{i}}\right)$ é Riemanniano, $\forall i \in\{1, \ldots, m\}$, então, $M$ é denominado um espaço-tempo de Robertson-Walker generalizado.

Note que, neste caso, a variedade correspondente é Lorentziana. Entretanto, estes não são os únicos produtos multiplamente torcidos Lorentzianos. Por exemplo, se $M=B \times{ }_{b} F$, onde $B$ é Riemanniano e $F$ é Lorentziano, então $M$ é Lorentziano $[5]$.

Considere um espaço-tempo de Robertson-Walker generalizado da forma $M=$ $I \times{ }_{b} F$, onde $F$ é uma variedade Riemanniana conexa tridimensional de curvatura seccional constante $S=-1,0$ ou 1 . Neste caso, $M$ é o espaço-tempo de RobertsonWalker clássico da cosmologia [22]. 
Neste capítulo, nas duas seções seguintes, discutem-se condições necessárias e/ou suficientes para que um espaço-tempo de Robertson-Walker generalizado seja uma variedade de Einstein ou possua curvatura escalar constante.

\subsection{Variedades de Einstein}

Definição 2.2 Uma variedade semi-Riemanniana $M$ é denominada de Einstein se, $\forall X, Y \in \mathscr{X}(M)$,

$$
\operatorname{Ric}(X, Y)=\lambda g(X, Y) \text {, onde } \lambda \in \mathcal{F}(M)
$$

Proposição 2.3 Se uma variedade semi-Riemanniana $M$, de dimensão $n$, é conexa $e$ de Einstein tal que $n \neq 2$, então $\lambda$ é uma função constante em $M$. Neste caso, a curvatura escalar é constante e dada por $S=\lambda n$.

Demonstração: Considere um ponto $p \in M$ arbitrário e um referencial geodésico em uma vizinhança $U$ de $p$, conforme a Proposição A.6. Então, em $p$, a segunda identidade de Bianchi (veja a Proposição A.7 no apêndice) pode ser escrita na forma

$$
D_{E_{i}}\left(R\left(E_{j}, E_{k}\right) E_{m}\right)(p)+D_{E_{j}}\left(R\left(E_{k}, E_{i}\right) E_{m}\right)(p)+D_{E_{k}}\left(R\left(E_{i}, E_{j}\right) E_{m}\right)(p)=0,
$$

$\forall i, j, k, m \in\{1, \ldots, n\}$, onde $\left\{E_{i}\right\}$ indica o referencial geodésico. Logo,

$$
g\left(D_{E_{i}}\left(R\left(E_{j}, E_{k}\right) E_{m}\right)+D_{E_{j}}\left(R\left(E_{k}, E_{i}\right) E_{m}\right)+D_{E_{k}}\left(R\left(E_{i}, E_{j}\right) E_{m}\right), E_{r}\right)_{p}=0
$$

$\forall i, j, k, m, r \in\{1, \ldots, n\}$

Note que

$$
\begin{aligned}
g\left(D_{E_{i}}\left(R\left(E_{j}, E_{k}\right) E_{m}\right), E_{r}\right)_{p} & =E_{i} g\left(R\left(E_{j}, E_{k}\right) E_{m}, E_{r}\right)_{p}-g\left(R\left(E_{j}, E_{k}\right) E_{m}, D_{E_{i}} E_{r}\right)_{p} \\
& =E_{i} g\left(R\left(E_{j}, E_{k}\right) E_{m}, E_{r}\right)_{p} \\
& =E_{i} g\left(\sum_{s=1}^{n} R_{m j k}^{s} E_{s}, E_{r}\right)_{p} \\
& =E_{i}\left(\sum_{s=1}^{n} g_{s_{r}} R_{m j k}^{s}\right)_{p}
\end{aligned}
$$


Portanto, em $p$,

$$
E_{i}\left(\sum_{s=1}^{n} g_{s_{r}} R_{m j k}^{s}\right)+E_{j}\left(\sum_{s=1}^{n} g_{s_{r}} R_{m k i}^{s}\right)+E_{k}\left(\sum_{s=1}^{n} g_{s_{r}} R_{m i j}^{s}\right)=0 .
$$

Note que, de acordo com a Proposição A.6, em $U, g_{i j}$ é uma matriz constante. Logo, $E_{i}\left(g_{j k}\right)=0, \forall i, j, k \in\{1, \ldots, n\}$.

Multiplique a última equação por $g^{i m} g^{j r}$ e some sobre todos os valores possíveis de $i, m, j$ e $r$. Em uma análise termo a termo, utilizando que $\operatorname{Ric}(X, Y)=\lambda g(X, Y)$,

$$
\begin{aligned}
\sum_{i, j, m, r, s=1}^{n} g^{i m} g^{j r} E_{i}\left(g_{s_{r}} R_{m j k}^{s}\right) & =\sum_{i, m=1}^{n} E_{i}\left(g^{i m} \lambda g_{m k}\right)=E_{k}(\lambda) \\
\sum_{i, j, m, r, s=1}^{n} g^{i m} g^{j r} E_{j}\left(g_{s_{r}} R_{m k i}^{s}\right) & =\sum_{i, j, m, r, s=1}^{n} g^{i m} g^{j r} E_{j}\left(g_{s_{m}} R_{r i k}^{s}\right) \\
& =\sum_{j, k=1}^{n} E_{j}\left(g^{j r} \lambda g_{r k}\right)=E_{k}(\lambda)
\end{aligned}
$$

onde utilizou-se que $g(R(x, y) z, w)=g(R(y, x) w, z), \forall x, y, z, w \in T_{p} M$;

$$
\begin{aligned}
\sum_{i, j, m, r, s=1}^{n} g^{i m} g^{j r} E_{k}\left(g_{s_{r}} R_{m i j}^{s}\right) & =-\sum_{i, m=1}^{n} E_{k}\left(g^{i m} \lambda g_{m i}\right) \\
& =-\sum_{i=1}^{n} \delta_{i}^{i} E_{k}(\lambda)=-n E_{k}(\lambda),
\end{aligned}
$$

onde utilizou-se que $g(R(x, y) z, w)=-g(R(y, x) z, w), \forall x, y, z, w \in T_{p} M$. Logo,

$$
(2-n) E_{k}(\lambda)=0, \forall k \in\{1, \ldots, n\}
$$

Portanto, se $n \neq 2, E_{k}(\lambda)=0$.

Se $M$ é conexa, tal que $n \neq 2$, isto implica que $\lambda$ é constante. Note que, se $M$ é unidimensional, o tensor de Riemann é identicamente nulo e, portanto, também, o tensor de Ricci (veja Proposição A.19).

Logo, se $n=1$,

$$
\operatorname{Ric}(X, Y)=0=0 g(X, Y), \forall X, Y \in \Re(M)
$$


ou seja, neste caso, $\lambda$ é nulo.

Note que

$$
S=\sum_{i, j=1}^{n} g^{i j} \operatorname{Ric}_{i j}=\sum_{i, j=1}^{n} \lambda g^{i j} g_{i j}=\lambda n .
$$

Proposição 2.4 Considere $B=I=\left(t_{1}, t_{2}\right)$ munido da métrica $g_{B}=-d t \otimes d t, \forall t \in$ I. Neste caso,

$$
\begin{aligned}
& \operatorname{grad}_{B} f=-\frac{\mathrm{d} f}{\mathrm{~d} t} \frac{\partial}{\partial t} \\
& H_{B}^{f}\left(\frac{\partial}{\partial t}, \frac{\partial}{\partial t}\right)=\frac{\mathrm{d}^{2} f}{\mathrm{~d} t^{2}} \quad e \\
& \Delta_{B} f=-\frac{\mathrm{d}^{2} f}{\mathrm{~d} t^{2}},
\end{aligned}
$$

$\forall f \in \mathcal{F}(B)$

Demonstração: (2.1) $g_{B}\left(\operatorname{grad}_{B} f, \frac{\partial}{\partial t}\right)=\frac{\partial f}{\partial t}=\frac{\mathrm{d} f}{\mathrm{~d} t} . \quad \operatorname{Logo}, \operatorname{grad}_{B} f=-\frac{\mathrm{d} f}{\mathrm{~d} t} \frac{\partial}{\partial t}$, pois $g_{B}\left(\frac{\partial}{\partial t}, \frac{\partial}{\partial t}\right)=-1$.

(2.2) $\frac{\partial}{\partial t}\left[g_{B}\left(\frac{\partial}{\partial t}, \frac{\partial}{\partial t}\right)\right]=\frac{\partial}{\partial t}(-1)=0=2 g_{B}\left(D_{\frac{\partial}{\partial t}}^{B} \frac{\partial}{\partial t}, \frac{\partial}{\partial t}\right)$. Logo, $D_{\frac{\partial}{\partial t}}^{B} \frac{\partial}{\partial t}=0$. Por definição,

$$
H_{B}^{f}\left(\frac{\partial}{\partial t}, \frac{\partial}{\partial t}\right)=\frac{\partial}{\partial t} \frac{\partial}{\partial t} f-\left(D_{\frac{\partial}{\partial t}}^{B} \frac{\partial}{\partial t}\right) f=\frac{\mathrm{d}^{2} f}{\mathrm{~d} t^{2}} .
$$

Portanto, $H_{B}^{f}=\frac{\mathrm{d}^{2} f}{\mathrm{~d} t^{2}} \mathrm{~d} t \otimes \mathrm{d} t$.

(2.3) Como $\Delta_{B} f=\sum_{i, j=1}^{1} g_{B}^{i j} H_{B i j}^{f}, \Delta_{B} f=-\frac{\mathrm{d}^{2} f}{\mathrm{~d} t^{2}}$.

Observação: A partir da Proposição 2.5, adota-se a seguinte convenção: funções na base e nas fibras e seus levantamentos são escritos da mesma forma. Por exemplo, $\overline{\operatorname{Ric}^{F_{i}}\left(V_{F_{i}}, U_{F_{i}}\right)}=\operatorname{Ric}^{F_{i}}\left(V_{F_{i}}, U_{F_{i}}\right)$. 
Proposição 2.5 Seja $M=I \times{ }_{b_{1}} F_{1} \times \cdots \times{ }_{b_{m}} F_{m}$ um espaço-tempo de RobertsonWalker generalizado. Dados $\frac{\partial}{\partial t} \in \mathscr{X}(I), f$ e $h \in \mathcal{F}(I)$ arbitrários, $V_{i}, U_{i} \in \mathcal{L}\left(F_{i}\right)$ arbitrários, $\forall i \in\{1, \ldots, m\}$. Sejam $V=\sum_{i=1}^{m} V_{i}$ e $U=\sum_{i=1}^{m} U_{i}$. Então,

$$
\begin{array}{r}
\operatorname{Ric}\left(f \frac{\bar{\partial}}{\partial t}+V, h \frac{\bar{\partial}}{\partial t}+U\right)=\sum_{i=1}^{m}\left\{\operatorname{Ric}^{F_{i}}\left(V_{F_{i}}, U_{F_{i}}\right)-f h s_{i} \frac{b_{i}^{\prime \prime}}{b_{i}}\right. \\
\left.+\left(b_{i} b_{i}^{\prime \prime}+\left(s_{i}-1\right)\left(b_{i}^{\prime}\right)^{2}+b_{i} b_{i}^{\prime} \sum_{k=1, k \neq i}^{m} s_{k} \frac{b_{k}^{\prime}}{b_{k}}\right) g_{F_{i}}\left(V_{F_{i}}, U_{F_{i}}\right)\right\},
\end{array}
$$

onde $b_{i}^{\prime}=\frac{\mathrm{d} b_{i}}{\mathrm{~d} t}, b_{i}^{\prime \prime}=\frac{\mathrm{d}^{2} b_{i}}{\mathrm{~d} t^{2}}, s_{i}=\operatorname{dim}\left(F_{i}\right), V_{i}=\overline{V_{F_{i}}}$ e $U_{i}=\overline{U_{F_{i}}}$.

Demonstração: É uma conseqüência imediata do fato de que Ric é linear e das Proposições 1.7, 2.4 e A.19.

Proposição 2.6 Seja $M=I \times{ }_{b_{1}} F_{1} \times \cdots \times{ }_{b_{m}} F_{m}$ um espaço-tempo de RobertsonWalker generalizado. Dados $p \in M$ e $V_{p} \in T_{p} M$ arbitrários, existem $\overline{V^{I}} \in \mathcal{L}(I)$ e $\overline{V^{F_{i}}} \in \mathcal{L}\left(F_{i}\right), \forall i \in\{1, \cdots, m\}$, tais que

$$
V_{p}=\overline{V^{I}}(p)+\overline{V^{F_{1}}}(p)+\cdots+\overline{V^{F_{m}}}(p)
$$

Demonstração: Seja $p=\left(q, f_{1}, \ldots, f_{m}\right) \in M$, onde $q \in I, f_{i} \in F_{i}, \forall i \in\{1, \ldots, m\}$. Como

$$
T_{p} M=T_{p} I \oplus T_{p} F_{1} \oplus \cdots \oplus T_{p} F_{m}
$$

resulta que $V_{p}=V_{p}^{I}+V_{p}^{F_{1}}+\cdots+V_{p}^{F_{m}}$, onde $V_{p}^{I} \in T_{p} I$ e $V_{p}^{F_{i}} \in T_{p} F_{i}, \forall i \in\{1, \ldots, m\}$.

Considere que

$$
\begin{aligned}
& \mathrm{d} \pi\left(V_{p}^{I}\right) \equiv V_{q}^{I}, \quad \mathrm{~d} \sigma_{i}\left(V_{p}^{I}\right)=0, \\
& \mathrm{~d} \pi\left(V_{p}^{F_{i}}\right)=0, \quad \mathrm{~d} \sigma_{j}\left(V_{p}^{F_{i}}\right)=0, \text { para } i \neq j \mathrm{e} \\
& \mathrm{d} \sigma_{i}\left(V_{p}^{F_{i}}\right) \equiv V_{f_{i}}^{F_{i}}, \quad \forall i, j \in\{1, \ldots, m\} .
\end{aligned}
$$


Portanto,

$$
V_{p}=\overline{V_{q}^{I}}+\overline{V_{f_{1}}^{F_{1}}}+\cdots+\overline{V_{f_{m}}^{F_{m}}}
$$

Dado $V_{q}^{I} \in T_{q} I$ arbitrário, conforme [22], existe $V^{I} \in \Re(I)$, tal que $V^{I}(q)=V_{q}^{I}$. Similarmente, dado $V_{f_{i}}^{F_{i}} \in T_{f_{i}} F_{i}$ arbitrário, existe $V^{F_{i}} \in \mathscr{X}\left(F_{i}\right)$, tal que $V^{F_{i}}\left(f_{i}\right)=$ $V_{f_{i}}^{F_{i}}, \forall i \in\{1, \ldots, m\}$. Logo,

$$
V_{p}=\overline{V^{I}}(p)+\overline{V^{F_{1}}}(p)+\cdots+\overline{V^{F_{m}}}(p)
$$

onde, de acordo com a definição de levantamento de campos vetoriais,

$$
\begin{aligned}
\overline{V^{I}}(p) & =\overline{V^{I}(q)} \mathrm{e} \\
\overline{V^{F_{i}}}(p) & =\overline{V^{F_{i}}\left(f_{i}\right)}, \quad \forall i \in\{1, \ldots, m\} .
\end{aligned}
$$

Note que $\overline{V^{I}}+\overline{V^{F_{1}}}+\cdots+\overline{V^{F_{m}}} \in \mathscr{X}(M)$, pois $\overline{V^{I}} \in \mathcal{L}(I) \subset \mathscr{X}(M)$ e $\overline{V^{F_{i}}} \in \mathcal{L}\left(F_{i}\right) \subset$ $\mathscr{X}(M), \forall i \in\{1, \ldots, m\}$.

Definição 2.7 Dada uma variedade de Einstein $M$ arbitrária, tal que Ric $=\lambda g$, então, a função $\lambda$ é denominada curvatura de Ricci.

Teorema 2.8 Seja $M=I \times{ }_{b_{1}} F_{1} \times \cdots \times{ }_{b_{m}} F_{m}$ um espaço-tempo de RobertsonWalker generalizado. Então $M$ é uma variedade de Einstein com curvatura de Ricci $\lambda$ se e somente se as seguintes condições são satisfeitas, $\forall i \in\{1, \ldots, m\}$ :

Cada fibra $\left(F_{i}, g_{F_{i}}\right)$ é uma variedade de Einstein com

curvatura de Ricci $\lambda_{F_{i}}, \forall i \in\{1, \ldots, m\}$,

$\sum_{i=1}^{m} s_{i} \frac{b_{i}^{\prime \prime}}{b_{i}}=\lambda e$

$\lambda_{F_{i}}+b_{i} b_{i}^{\prime \prime}+\left(s_{i}-1\right)\left(b_{i}^{\prime}\right)^{2}+b_{i} b_{i}^{\prime} \sum_{k=1, k \neq i}^{m} s_{k} \frac{b_{k}^{\prime}}{b_{k}}=\lambda b_{i}^{2}$. 
Demonstração: (a) Suponha que $M$ é uma variedade de Einstein com curvatura de Ricci $\lambda$. Considere a Proposição $2.5 \operatorname{com} V=U=0$. Resulta na equação (2.5).

Considere a Proposição $2.5 \mathrm{com} f, h=0$ e $V=V_{j}, U=U_{j}$, onde $j \in\{1, \ldots, m\}$. Como $g\left(V_{j}, U_{j}\right)=b_{j}^{2} g_{F_{j}}\left(V_{F_{j}}, U_{F_{j}}\right)$, obtém-se (2.4) e, conseqüentemente, (2.6).

(b) Suponha que as condições (2.4), (2.5), (2.6) são satisfeitas.

Deseja-se verificar que $\operatorname{Ric}(V, W)=\lambda g(V, W), \forall V, W \in \mathfrak{X}(M)$ onde $\lambda \in \mathcal{F}(M)$. Ou seja,

$$
[\operatorname{Ric}(V, W)](p)=\lambda(p)[g(V, W)](p), \forall p \in M,
$$

o que equivale a

$$
\operatorname{Ric}\left(V_{p}, W_{p}\right)=\lambda(p) g\left(V_{p}, W_{p}\right),
$$

onde $V_{p}=V(p)$ e $W_{p}=W(p)$.

Conforme a Proposição A.18,

$$
\begin{aligned}
\operatorname{Ric}\left(V_{p}, W_{p}\right) & =[\operatorname{Ric}(X, Y)](p) \mathrm{e} \\
g\left(V_{p}, W_{p}\right) & =[g(X, Y)](p),
\end{aligned}
$$

$\forall X, Y \in \mathfrak{X}(M)$ tais que $X(p)=V_{p}$ e $Y(p)=W_{p}$.

Ou seja, de acordo com a Proposição 2.6, é suficiente mostrar que

$$
\begin{aligned}
& {\left[\operatorname{Ric}\left(\overline{V^{I}}+\overline{V^{F_{1}}}+\cdots+\overline{V^{F_{m}}}, \overline{W^{I}}+\overline{W^{F_{1}}}+\cdots+\overline{W^{F_{m}}}\right)\right](p)} \\
& =\lambda(p)\left[g\left(\overline{V^{I}}+\overline{V^{F_{1}}}+\cdots+\overline{V^{F_{m}}}, \overline{W^{I}}+\overline{W^{F_{1}}}+\cdots+\overline{W^{F_{m}}}\right)\right](p),
\end{aligned}
$$

$\forall \overline{V^{I}}, \overline{W^{I}} \in \mathcal{L}(I), \overline{V^{F_{i}}}, \overline{W^{F_{i}}} \in \mathcal{L}\left(F_{i}\right), \forall i \in\{1, \ldots, m\}, \forall p \in M$, onde $\lambda \in \mathcal{F}(M)$.

Utilizando a Proposição 2.5 e as condições (2.4), (2.5) e (2.6) deste teorema,

$$
\begin{aligned}
\operatorname{Ric}\left(f \frac{\bar{\partial}}{\partial t}+V, h \frac{\bar{\partial}}{\partial t}+U\right) & =\sum_{i=1}^{m}\left\{\lambda_{F_{i}} g_{F_{i}}\left(V_{F_{i}}, U_{F_{i}}\right)+\left(\lambda b_{i}^{2}-\lambda_{F_{i}}\right) g_{F_{i}}\left(V_{F_{i}}, U_{F_{i}}\right)\right\}-f h \lambda \\
& =\lambda g(V, U)+\lambda f h g\left(\frac{\bar{\partial}}{\partial t}, \frac{\bar{\partial}}{\partial t}\right) \\
& =\lambda g\left(f \frac{\bar{\partial}}{\partial t}+V, h \frac{\bar{\partial}}{\partial t}+U\right) .
\end{aligned}
$$


Como $f, h, V$ e $U$ são arbitrários, resulta que $M$ é uma variedade de Einstein com curvatura de Ricci $\lambda$.

Proposição 2.9 A condição (2.6) do Teorema 2.8 pode ser colocada, equivalentemente, nas formas

$$
\frac{\lambda_{F_{i}}}{b_{i}^{2}}+\frac{1}{s_{i}} \frac{\left(b_{i}^{s_{i}}\right)^{\prime \prime}}{b_{i}^{s_{i}}}+\frac{b_{i}^{\prime}}{b_{i}} \sum_{k=1, k \neq i}^{m} s_{k} \frac{b_{k}^{\prime}}{b_{k}}=\lambda
$$

ou

$$
\frac{\lambda_{F_{i}}}{b_{i}^{2}}+\frac{b_{i}^{\prime \prime}}{b_{i}}-\frac{\left(b_{i}^{\prime}\right)^{2}}{b_{i}^{2}}+\frac{b_{i}^{\prime}}{b_{i}} \sum_{k=1}^{m} s_{k} \frac{b_{k}^{\prime}}{b_{k}}=\lambda,
$$

$\forall i \in\{1, \ldots, m\}$, onde $s_{i}=\operatorname{dim}\left(F_{i}\right) \neq 0$.

Demonstração: (2.7) É suficiente demonstrar que

$$
\frac{b_{i}^{\prime \prime}}{b_{i}}+\left(s_{i}-1\right) \frac{\left(b_{i}^{\prime}\right)^{2}}{b_{i}^{2}}=\frac{1}{s_{i}} \frac{\left(b_{i}^{s_{i}}\right)^{\prime \prime}}{b_{i}^{s_{i}}}
$$

o que é imediato, pois $\left(b_{i}^{s_{i}}\right)^{\prime \prime}=s_{i}\left(s_{i}-1\right) b_{i}^{s_{i}-2}\left(b_{i}^{\prime}\right)^{2}+b_{i}^{s_{i}-1} b_{i}^{\prime \prime} s_{i}$.

(2.8) Segue do fato que $\left(s_{i}-1\right) \frac{\left(b_{i}^{\prime}\right)^{2}}{b_{i}^{2}}=-\frac{\left(b_{i}^{\prime}\right)^{2}}{b_{i}^{2}}+\frac{b_{i}^{\prime}}{b_{i}} s_{i} \frac{b_{i}^{\prime}}{b_{i}}$.

\subsection{Curvatura escalar constante}

O resultado a seguir encontra-se na referência [12]:

Proposição 2.10 Seja $M$ uma variedade semi-Riemanniana e $L$ um operador atuando nas funções $f \in \mathcal{F}(M)$ tais que $f(p)>0, \forall p \in M$, definido por

$$
L f=\sum_{i=1}^{k} r_{i} \frac{\Delta\left(f^{a_{i}}\right)}{f^{a_{i}}}
$$

onde $k \in \mathbb{Z}, k \geq 1, r_{i}, a_{i} \in \mathbb{R}$. Definindo $\psi \equiv \sum_{i=1}^{k} r_{i} a_{i}$ e $\chi \equiv \sum_{i=1}^{k} r_{i} a_{i}^{2}$, então,

$$
L f=\frac{(\chi-\psi)}{f^{2}} g(\operatorname{grad} f, \operatorname{grad} f)+\psi \frac{\Delta f}{f} .
$$


Se $\psi \neq 0$ e $\chi \neq 0$, definindo $\alpha=\frac{\psi}{\chi}$ e $\beta=\frac{\psi^{2}}{\chi}$, então

$$
L f=\beta \frac{\Delta\left(f^{1 / \alpha}\right)}{f^{1 / \alpha}} .
$$

Demonstração: (2.9) Utilizando que

$$
\frac{\Delta\left(f^{a_{i}}\right)}{f^{a_{i}}}=a_{i}\left[\frac{\left(a_{i}-1\right)}{f^{2}} g(\operatorname{grad} f, \operatorname{grad} f)+\frac{\Delta f}{f}\right],
$$

conforme a Proposição A.14, resulta a identidade (2.9).

(2.10) Similarmente,

$$
\begin{aligned}
\frac{\beta \Delta\left(f^{1 / \alpha}\right)}{f^{1 / \alpha}} & =\beta \frac{1}{\alpha}\left[\left(\frac{1}{\alpha}-1\right) \frac{g(\operatorname{grad} f, \operatorname{grad} f)}{f^{2}}+\frac{\Delta f}{f}\right] \\
& =\sum_{i=1}^{k} r_{i} a_{i}\left[\left(\frac{\sum_{i=1}^{k} r_{i} a_{i}^{2}}{\sum_{i=1}^{k} r_{i} a_{i}}-1\right) \frac{g(\operatorname{grad} f, \operatorname{grad} f)}{f^{2}}+\frac{\Delta f}{f}\right] \\
& =\frac{(\chi-\psi)}{f^{2}} g(\operatorname{grad} f, \operatorname{grad} f)+\psi \frac{\Delta f}{f} \\
& =L f
\end{aligned}
$$

utilizando (2.9).

Proposição 2.11 Seja $M=I \times{ }_{b_{1}} F_{1} \times \cdots \times{ }_{b_{m}} F_{m}$ um espaço-tempo de RobertsonWalker generalizado. Então, a curvatura escalar admite as seguintes expressões:

$$
\begin{aligned}
S & =\sum_{i=1}^{m}\left\{2 s_{i} \frac{b_{i}^{\prime \prime}}{b_{i}}+\frac{S_{F_{i}}}{b_{i}^{2}}+s_{i}\left(s_{i}-1\right) \frac{\left(b_{i}^{\prime}\right)^{2}}{b_{i}^{2}}+\sum_{k=1, k \neq i}^{m} s_{k} s_{i} \frac{b_{i}^{\prime} b_{k}^{\prime}}{b_{i} b_{k}}\right\}, \\
S & =\sum_{i=1}^{m}\left[s_{i} \frac{b_{i}^{\prime \prime}}{b_{i}}+s_{i}\left(\frac{b_{i}^{\prime}}{b_{i}}\right)^{\prime}+\frac{S_{F_{i}}}{b_{i}^{2}}\right]+\left(\sum_{i=1}^{m} \frac{s_{i} b_{i}^{\prime}}{b_{i}}\right)^{2}, \\
S & =\sum_{i=1}^{m}\left\{s_{i} \frac{b_{i}^{\prime \prime}}{b_{i}}+\frac{\left(b_{i}^{s_{i}}\right)^{\prime \prime}}{b_{i}^{s_{i}}}+\frac{S_{F_{i}}}{b_{i}^{2}}+\sum_{k=1, k \neq i}^{m} s_{k} s_{i} \frac{b_{i}^{\prime} b_{k}^{\prime}}{b_{i} b_{k}}\right\}, \\
S & =\sum_{i=1}^{m}\left\{\frac{4 s_{i}}{\left(s_{i}+1\right)} \frac{\left(b_{i}^{\left(s_{i}+1\right) / 2}\right)^{\prime \prime}}{b_{i}^{\left(s_{i}+1\right) / 2}}+\frac{S_{F_{i}}}{b_{i}^{2}}+\sum_{k=1, k \neq i}^{m} s_{k} s_{i} \frac{b_{i}^{\prime} b_{k}^{\prime}}{b_{i} b_{k}}\right\} e \\
S & =\sum_{i=1}^{m}\left\{\frac{4 s_{i}}{\left(s_{i}+1\right)} \frac{\psi_{i}^{\prime \prime}}{\psi_{i}}+\frac{S_{F_{i}}}{\psi_{i}^{4 /\left(s_{i}+1\right)}}+\sum_{k=1, k \neq i}^{m} s_{k} s_{i} \frac{\left(\psi_{i}^{2 /\left(s_{i}+1\right)}\right)^{\prime}}{\psi_{i}^{2 /\left(s_{i}+1\right)}} \frac{\left(\psi_{k}^{2 /\left(s_{k}+1\right)}\right)^{\prime}}{\left.\psi_{k}^{2 /\left(s_{k}+1\right)}\right\},}\right.
\end{aligned}
$$

onde $\psi_{i}=b_{i}^{\left(s_{i}+1\right) / 2}$. 
Demonstração: (2.11) É uma conseqüência direta das Proposições 1.8, 2.4 e A.19.

(2.12) É uma conseqüência do fato de que $\left(\frac{b_{i}^{\prime}}{b_{i}}\right)^{\prime}=\frac{b_{i}^{\prime \prime}}{b_{i}}-\frac{\left(b_{i}^{\prime}\right)^{2}}{b_{i}^{2}}$.

(2.13) Segue do fato que $\frac{\left(b_{i}^{s_{i}}\right)^{\prime \prime}}{b_{i}^{s_{i}}}=\frac{s_{i} b_{i}^{\prime \prime}}{b_{i}}+s_{i}\left(s_{i}-1\right) \frac{\left(b_{i}^{\prime}\right)^{2}}{b_{i}^{2}}$

(2.14) $\frac{s_{i} b_{i}^{\prime \prime}}{b_{i}}+\frac{\left(b_{i}^{s_{i}}\right)^{\prime \prime}}{b_{i}^{s_{i}}}=-s_{i} \frac{\Delta_{B} b_{i}}{b_{i}}-\frac{\Delta_{B}\left(b_{i}^{s_{i}}\right)}{b_{i}^{s_{i}}}$.

Utilizando a Proposição 2.10, equação (2.10) e notando que $\psi=-2 s_{i} \neq 0$, $\chi=-\left(s_{i}^{2}+s_{i}\right) \neq 0, \alpha=\frac{2}{s_{i}+1}$ e $\beta=\frac{-4 s_{i}}{s_{i}+1}$, resulta que

$$
\frac{s_{i} b_{i}^{\prime \prime}}{b_{i}}+\frac{\left(b_{i}^{s_{i}}\right)^{\prime \prime}}{b_{i}^{s_{i}}}=-\frac{4 s_{i}}{\left(s_{i}+1\right)} \frac{\Delta_{B}\left(b_{i}^{\left(s_{i}+1\right) / 2}\right)}{b_{i}^{\left(s_{i}+1\right) / 2}}=\frac{4 s_{i}}{\left(s_{i}+1\right)} \frac{\left(b_{i}^{\left(s_{i}+1\right) / 2}\right)^{\prime \prime}}{b_{i}^{\left(s_{i}+1\right) / 2}} .
$$

Substituindo esta última expressão em (2.13), resulta (2.14).

(2.15) Partindo de (2.14) e fazendo a substituição $\psi_{i}=b_{i}^{\left(s_{i}+1\right) / 2}$, resulta $(2.15)$.

Proposição 2.12 Seja $M=I \times{ }_{b_{1}} F_{1} \times \cdots \times{ }_{b_{m}} F_{m}$ um espaço-tempo de RobertsonWalker generalizado. Se a curvatura escalar em $M$ é constante, então, em cada fibra $F_{i}$ a curvatura escalar é constante, $\forall i \in\{1, \ldots, m\}$.

Demonstração: Da Proposição 2.11, equação (2.11), resulta que

$$
\begin{aligned}
S_{F_{j}}= & b_{j}^{2}\left\{S-\sum_{i=1}^{m}\left(2 s_{i} \frac{b_{i}^{\prime \prime}}{b_{i}}+s_{i}\left(s_{i}-1\right) \frac{\left(b_{i}^{\prime}\right)^{2}}{b_{i}^{2}}+\sum_{k=1, k \neq i}^{m} s_{k} s_{i} \frac{b_{i}^{\prime} b_{k}^{\prime}}{b_{i} b_{k}}\right)\right\} \\
& -b_{j}^{2} \sum_{i=1, i \neq j}^{m} \frac{S_{F_{i}}}{b_{i}^{2}}, \forall j \in\{1, \ldots, m\} .
\end{aligned}
$$

Construindo um sistema de coordenadas em $M$ a partir das coordenadas na base e nas fibras, resulta que o lado esquerdo da equação é função das coordenadas na fibra $F_{j}$, enquanto que o lado direito não depende das coordenadas da fibra $F_{j}$. Como o lado direito é igual ao lado esquerdo, resulta que $S_{F_{j}}$ não depende das 
coordenadas em $F_{j}$. Por definição, $S_{F_{j}}$ não depende das coordenadas na base e nas outras fibras. Logo, $S_{F_{j}}$ é constante.

Deseja-se, agora, mostrar que a condição mencionada na Proposição 2.12 para que um espaço-tempo de Robertson-Walker generalizado possua curvatura escalar constante é necessária, porém, não é suficiente.

Exemplo 2.13 Considere o espaço-tempo de Robertson-Walker generalizado dado por $M=(0, \infty) \times_{\varphi} \mathbb{R} \times{ }_{\varphi} \mathbb{R} \times{ }_{\varphi} \mathbb{R}$, onde $\varphi(t)=t, \forall t \in(0, \infty)$. Conforme a Proposição 2.11 , equação (2.11),

$$
S=\sum_{i=1}^{3} \sum_{k=1, k \neq i}^{3} \frac{1}{t^{2}}=\frac{1}{t^{2}} \sum_{i=1}^{3}(2)=\frac{6}{t^{2}} \text {. }
$$

Portanto, neste caso, a curvatura escalar de cada fibra é constante (igual a zero), mas a curvatura escalar em $M$ não é constante. 



\section{Capítulo 3}

\section{Espaços-tempo de Kasner generalizados}

Os principais resultados deste capítulo estão contidos na referência [13].

Definição 3.1 Um espaço-tempo de Kasner generalizado é um espaço-tempo de Robertson-Walker generalizado da forma $M=I \times \times_{\varphi^{p_{1}}} F_{1} \times \cdots \times \times_{\varphi^{p_{m}}} F_{m}$, onde $\varphi$ : $I \rightarrow(0, \infty) \in \mathcal{F}(I)$ e $p_{i} \in \mathbb{R}, \forall i \in\{1, \ldots, m\}$.

O espaço-tempo de Kasner usual ou clássico, conforme [21], corresponde ao caso $I=(0, \infty), m=3, F_{1}=F_{2}=F_{3}=\mathbb{R}, \varphi(t)=t, \forall t \in I$ e $p_{1}+p_{2}+p_{3}=$ $\left(p_{1}\right)^{2}+\left(p_{2}\right)^{2}+\left(p_{3}\right)^{2}=1$.

Na primeira seção deste capítulo, os resultados do capítulo anterior são aplicados aos espaços-tempo de Kasner generalizados. Nas seções seguintes, é discutida a classificação dos espaços-tempo de Kasner generalizados de dimensão quatro que sejam, particularmente, variedades de Einstein ou possuam curvatura escalar constante.

\subsection{Espaços-tempo de dimensão arbitrária}

Definição 3.2 Dado um espaço-tempo de Kasner generalizado $M=I \times{ }_{\varphi^{p_{1}}} F_{1} \times$ $\cdots \times_{\varphi^{p_{m}}} F_{m}$, é conveniente definir as variáveis $\zeta=\sum_{i=1}^{m} s_{i} p_{i}$ e $\eta=\sum_{i=1}^{m} s_{i} p_{i}^{2}$, onde $s_{i}=\operatorname{dim}\left(F_{i}\right)$. Note que $\zeta \neq 0$ implica em $\eta \neq 0$. 
Proposição 3.3 Seja $M=I \times_{\varphi^{p_{1}}} F_{1} \times \cdots \times_{\varphi^{p_{m}}} F_{m}$ um espaço-tempo de Kasner generalizado. Então, $M$ é uma variedade de Einstein com curvatura de Ricci $\lambda$ se $e$ somente se, $\forall i \in\{1, \ldots, m\}$,

Cada fibra $F_{i}$ é uma variedade de Einstein com curvatura de Ricci $\lambda_{F_{i}}$, (3.1)

$$
\begin{aligned}
& \lambda=\sum_{i=1}^{m} s_{i} \frac{\left(\varphi^{p_{i}}\right)^{\prime \prime}}{\varphi^{p_{i}}}=(\eta-\zeta) \frac{\left(\varphi^{\prime}\right)^{2}}{\varphi^{2}}+\frac{\zeta \varphi^{\prime \prime}}{\varphi} e \\
& \frac{\lambda_{F_{i}}}{\varphi^{2 p_{i}}}+p_{i}\left[(\zeta-1) \frac{\left(\varphi^{\prime}\right)^{2}}{\varphi^{2}}+\frac{\varphi^{\prime \prime}}{\varphi}\right]=\lambda .
\end{aligned}
$$

Demonstração: É uma conseqüência imediata do Teorema 2.8 e da Definição 3.2.

Proposição 3.4 As condições (3.2) e (3.3) na Proposição 3.3 podem ser colocadas, respectivamente, nas seguintes formas, se $\zeta \neq 0$ :

$$
\begin{aligned}
& \lambda=\frac{\zeta^{2}}{\eta} \frac{\left(\varphi^{\eta / \zeta}\right)^{\prime \prime}}{\varphi^{\eta / \zeta}} \quad e \\
& \frac{\lambda_{F_{i}}}{\varphi^{2 p_{i}}}+\frac{p_{i}}{\zeta} \frac{\left(\varphi^{\zeta}\right)^{\prime \prime}}{\varphi^{\zeta}}=\lambda, \quad \forall i \in\{1, \ldots, m\} .
\end{aligned}
$$

Demonstração: Desenvolvendo as derivadas de segunda ordem nas expressões (3.4) e (3.5) resulta nas condições (3.2) e (3.3) da proposição anterior.

Observação: Note que o espaço-tempo de Kasner clássico corresponde a $\zeta=\eta=1$, $\lambda_{F_{1}}=\lambda_{F_{2}}=\lambda_{F_{3}}=0$. Portanto, conforme a Proposição 3.3, tal variedade é "Ricciflat", ou seja, o seu tensor de Ricci é identicamente nulo.

Proposição 3.5 Seja $M=I \times{ }_{\varphi^{p_{1}}} F_{1} \times \cdots \times \times_{\varphi^{p_{m}}} F_{m}$ um espaço-tempo de Kasner generalizado. Então, se $M$ possui curvatura escalar constante $S$,

Cada fibra $F_{i}$ possui curvatura escalar constante $S_{F_{i}}, \forall i \in\{1, \ldots, m\}$ e (3.6)

$$
S=2 \zeta \frac{\varphi^{\prime \prime}}{\varphi}+[(\zeta-2) \zeta+\eta] \frac{\left(\varphi^{\prime}\right)^{2}}{\varphi^{2}}+\sum_{i=1}^{m} \frac{S_{F_{i}}}{\varphi^{2 p_{i}}} .
$$


Demonstração: A condição (3.6) é a aplicação da Proposição 2.12 a este caso particular. A equação (3.7) resulta da equação (2.11) da Proposição 2.11, notando que $b_{i}=\varphi^{p_{i}}$ e

$$
\begin{aligned}
& \sum_{i=1}^{m}\left(2 s_{i} p_{i}\left(p_{i}-1\right)+s_{i}\left(s_{i}-1\right) p_{i}^{2}+\sum_{k=1, k \neq i}^{m} s_{i} p_{i} s_{k} p_{k}\right) \\
& =-2 \zeta+\eta+\sum_{i=1}^{m} s_{i} p_{i}\left(s_{i} p_{i}+\sum_{k=1, k \neq i}^{m} s_{k} p_{k}\right) \\
& =-2 \zeta+\eta+\zeta^{2}=(\zeta-2) \zeta+\eta .
\end{aligned}
$$

Proposição 3.6 Se $\zeta \neq 0$, a equação (3.7) na Proposição 3.5 pode ser colocada, de modo equivalente, nas formas:

$$
\begin{aligned}
S & =\frac{4 \zeta^{2}}{\zeta^{2}+\eta} \frac{\left(\varphi^{\left(\zeta^{2}+\eta\right) /(2 \zeta)}\right)^{\prime \prime}}{\varphi^{\left(\zeta^{2}+\eta\right) /(2 \zeta)}}+\sum_{i=1}^{m} \frac{S_{F_{i}}}{\varphi^{2 p_{i}}} \quad e \\
S & =\frac{4 \zeta^{2}}{\zeta^{2}+\eta} \frac{\mu^{\prime \prime}}{\mu}+\sum_{i=1}^{m} \frac{S_{F_{i}}}{\mu^{\left(4 \zeta p_{i} /\left(\zeta^{2}+\eta\right)\right)}}, \quad \text { onde } \mu=\varphi^{\left(\zeta^{2}+\eta\right) /(2 \zeta)} .
\end{aligned}
$$

Demonstração: (3.8) Em primeiro lugar, note que $\zeta \neq 0$ implica em $\eta \neq 0$ e, portanto, $(\zeta-1)^{2}+\eta \neq 0$. Utilizando o fato de que $\forall f \in \mathcal{F}(I)$, tal que $f>0$, $s \in \mathbb{R}$, conforme a Proposição A.14,

$$
\Delta_{I}\left(f^{s}\right)=s\left[(s-1) f^{s-2} g_{I}\left(\operatorname{grad}_{I} f, \operatorname{grad}_{I} f\right)+f^{s-1} \Delta_{I} f\right]
$$

e a Proposição 2.4, resulta que, substituíndo $f=\varphi$ e $s=(\zeta-1)^{2}+\eta$ na identidade acima,

$$
\frac{1}{(\zeta-1)^{2}+\eta} \frac{\left(\varphi^{(\zeta-1)^{2}+\eta}\right)^{\prime \prime}}{\varphi^{(\zeta-1)^{2}+\eta}}=[(\zeta-2) \zeta+\eta]\left(\frac{\varphi^{\prime}}{\varphi}\right)^{2}+\frac{\varphi^{\prime \prime}}{\varphi}
$$

Logo, utilizando (3.7),

$$
\begin{aligned}
S & =(2 \zeta-1) \frac{\varphi^{\prime \prime}}{\varphi}+\frac{1}{(\zeta-1)^{2}+\eta} \frac{\left(\varphi^{(\zeta-1)^{2}+\eta}\right)^{\prime \prime}}{\varphi^{(\zeta-1)^{2}+\eta}}+\sum_{i=1}^{m} \frac{S_{F_{i}}}{\varphi^{2 p_{i}}} \\
& =-(2 \zeta-1) \frac{\Delta_{I}(\varphi)}{\varphi}-\frac{1}{(\zeta-1)^{2}+\eta} \frac{\Delta_{I}\left(\varphi^{(\zeta-1)^{2}+\eta}\right)}{\varphi^{(\zeta-1)^{2}+\eta}}+\sum_{i=1}^{m} \frac{S_{F_{i}}}{\varphi^{2 p_{i}}}
\end{aligned}
$$


Os dois primeiros termos na expressão anterior podem ser reescritos utilizando a Proposição 2.10. Note que $\psi=-2 \zeta \neq 0$ e $\chi=-\left(\zeta^{2}+\eta\right) \neq 0$. Como $\alpha=\frac{2 \zeta}{\zeta^{2}+\eta}$ e $\beta=-\frac{4 \zeta^{2}}{\zeta^{2}+\eta}$, a equação (2.10) implica na equação (3.8).

(3.9) A mudança de variável $\mu=\varphi^{\left(\zeta^{2}+\eta\right) /(2 \zeta)}$ aplicada à equação (3.8) resulta na equação (3.9).

Exemplo 3.7 Seja $M=I \times_{\varphi^{p_{1}}} F_{1} \times \cdots \times_{\varphi^{p_{m}}} F_{m}$ um espaço-tempo de Kasner generalizado tal que $S_{F_{i}}=0, \forall i \in\{1, \ldots, m\}$ e $\zeta \neq 0$. Neste caso, a equação (3.9) da Proposição 3.6 resulta em

$$
\frac{4 \zeta^{2}}{\zeta^{2}+\eta} \mu^{\prime \prime}=\mu S
$$

A solução geral desta equação diferencial linear de segunda ordem é dada por

$$
\mu(t)=\left\{\begin{array}{l}
A \exp \left(i t \sqrt{-S \frac{\zeta^{2}+\eta}{4 \zeta^{2}}}\right)+B \exp \left(-i t \sqrt{-S \frac{\zeta^{2}+\eta}{4 \zeta^{2}}}\right), \text { se } S<0, \\
A t+B, \text { se } S=0, \\
A \exp \left(t \sqrt{S \frac{\zeta^{2}+\eta}{4 \zeta^{2}}}\right)+B \exp \left(-t \sqrt{S \frac{\zeta^{2}+\eta}{4 \zeta^{2}}}\right), \text { se } S>0,
\end{array}\right.
$$

com constantes $A, B \in \mathbb{R}$ escolhidas de tal modo que $\mu(t)>0, \forall t \in I$.

Se $\zeta=0$ e se $M$ possui curvatura escalar constante, então, conforme a Proposição 3.5

$$
S=\eta\left(\frac{\varphi^{\prime}}{\varphi}\right)^{2} \text {, onde } \varphi(t)>0, \forall t \in I .
$$

Note que $\eta \geq 0$, por definição. Se $\eta=0$, então $S=0$ e $M$ é o produto direto usual $M=I \times F_{1} \times \cdots \times F_{m}$, pois $p_{i}=0, \forall i \in\{1, \ldots, m\}$, onde $S_{F_{i}}=0$.

Se $\eta \neq 0$, note que $S=\eta\left(\frac{\varphi^{\prime}}{\varphi}\right)^{2}$ implica que $S \geq 0$. Logo,

$$
\left(\varphi \sqrt{\frac{S}{\eta}}+\varphi^{\prime}\right)\left(\varphi \sqrt{\frac{S}{\eta}}-\varphi^{\prime}\right)=0 .
$$


Soluções desta equação são dadas por

$$
\varphi(t)=C \exp \left( \pm t \sqrt{\frac{S}{\eta}}\right)
$$

onde $C \in \mathbb{R}, C>0$.

Observação: Note que o espaço-tempo de Kasner usual ou clássico possui curvatura escalar $S=0$. Como a equação (3.7) na Proposição 3.5 é válida independentemente do fato de $S$ ser constante ou não, $\zeta=\eta=1, \varphi^{\prime \prime}=0$ e $S_{F_{1}}=S_{F_{2}}=S_{F_{3}}=0$, resulta que $S=0$. Isto é uma conseqüência do fato que dada uma variedade semi-Riemanniana conexa arbitrária $M$ que seja uma variedade de Einstein com $\operatorname{dim}(M) \geq 3$ e curvatura de Ricci $\lambda$ (necessariamente constante), então, como $\lambda \operatorname{dim}(M)=S$, conforme a Proposição 2.3, segue que $S$ é necessariamente constante. Como $\lambda=0$ para o espaço-tempo clássico de Kasner, cuja dimensão é igual a quatro, segue que $S=4 \lambda=0$.

\subsection{Espaços-tempo de dimensão quatro}

O objetivo destas seções finais é discutir a classificação dos espaços-tempo de Kasner generalizados de dimensão quatro que possuam curvatura escalar constante ou que sejam variedades de Einstein. Para isto a seguinte definição é conveniente:

Definição 3.8 Seja $M=I \times_{b_{1}} F_{1} \times \cdots \times_{b_{m}} F_{m}$ um espaço-tempo de RobertsonWalker generalizado. Então:

(a) $M$ é dito do tipo I se $m=1$ e $\operatorname{dim}(F)=3$.

(b) $M$ é dito do tipo II se $m=2, \operatorname{dim}\left(F_{1}\right)=1$ e $\operatorname{dim}\left(F_{2}\right)=2$.

(c) $M$ é dito do tipo III se $m=3 \operatorname{dim}\left(F_{1}\right)=\operatorname{dim}\left(F_{2}\right)=\operatorname{dim}\left(F_{3}\right)=1$. 
A análise de variedades do tipo I com curvatura escalar constante pode ser encontrada no artigo de P. E. Ehrlich, Y. Jung e S. Kim [14].

De acordo com a Proposição 2.11, a curvatura escalar de $M$ do tipo I é dada por

$$
S=6\left(\frac{b^{\prime \prime}}{b}+\left(\frac{b^{\prime}}{b}\right)^{2}\right)+\frac{S_{F}}{b^{2}} .
$$

Definindo $v: I \rightarrow \mathbb{R}$, por $b(t)=\sqrt{v(t)}$, segue que

$$
S=\frac{S_{F}}{v}+\frac{3 v^{\prime \prime}}{v}
$$

Note que $v(t)>0, \forall t \in I$, pois $v(t)=(b(t))^{2}$ e $b(t)>0, \forall t \in I$.

Portanto, o problema de encontrar $M$ do tipo I tal que $S$ é constante é equivalente ao de encontrar soluções para a equação diferencial

$$
v^{\prime \prime}-\frac{S v}{3}+\frac{S_{F}}{3}=0
$$

tais que $v(t)>0, \forall t \in I$, onde $S_{F}$ é uma constante, de acordo com a Proposição 2.12 .

A solução geral da equação diferencial acima é dada por:

$$
\begin{aligned}
& v(t)=C_{1} \exp \left(t \sqrt{\frac{S}{3}}\right)+C_{2} \exp \left(-t \sqrt{\frac{S}{3}}\right)+\frac{S_{F}}{S}, \text { se } S>0 \\
& v(t)=-\frac{S_{F}}{6} t^{2}+C_{1} t+C_{2}, \text { se } S=0 \mathrm{e} \\
& v(t)=C_{1} \operatorname{sen}\left(t \sqrt{-\frac{S}{3}}\right)+C_{2} \cos \left(t \sqrt{-\frac{S}{3}}\right)+\frac{S_{F}}{S}, \text { se } S<0
\end{aligned}
$$

onde $C_{1}, C_{2} \in \mathbb{R}$ devem ser escolhidos de modo a fazer que, se possível, $v(t)>0$, $\forall t \in I$. Em relação a este último ponto, note que isto depende dos valores de $S, S_{F}$ e dos parâmetros $t_{1}, t_{2}$ do intervalo $I=\left(t_{1}, t_{2}\right)$. Para uma análise mais detalhada desta questão, consulte [14].

Um espaço-tempo de Kasner generalizado do tipo I consiste de $M=I \times \times_{\varphi^{p}} F$, onde $\varphi \in \mathcal{F}(I)$ e $p \in \mathbb{R}$. Logo, o problema de encontrar tais variedades com curvatura escalar constante consiste em considerar $\varphi^{p}=b=\sqrt{v}$ na discussão acima. 
A referência para variedades do tipo I que sejam de Einstein é o artigo de L. J. Alías, A. Romero e M. Sánchez [1].

De acordo com o Teorema 2.8, se $M=I \times_{b} F$ é uma variedade de Einstein, onde $F$ e $M$ são variedades conexas, então,

$$
\frac{b^{\prime \prime}}{b}=\frac{\lambda}{3} \text { e } \lambda_{F}+b b^{\prime \prime}+2\left(b^{\prime}\right)^{2}=\lambda b^{2}
$$

onde $\operatorname{dim}(F)=3$ e as constantes $\lambda, \lambda_{F}$ são as curvaturas de Ricci de $M$ e $F$, respectivamente. O seguinte sistema é equivalente ao sistema anterior:

$$
\frac{b^{\prime \prime}}{b}=\frac{\lambda}{3} \text { e } \frac{2 \lambda}{3}=\frac{\lambda_{F}+2\left(b^{\prime}\right)^{2}}{b^{2}}
$$

A primeira equação acima é linear e, portanto, as soluções do sistema podem ser encontradas. A tabela a seguir pode ser encontrada em [1]:

\begin{tabular}{|c|c|c|}
\hline$\lambda>0$ & $\lambda_{F}>0$ & $b(t)=a \exp \left(t \sqrt{\frac{\lambda}{3}}\right)+\frac{3}{8} \frac{\lambda_{F}}{a \lambda} \exp \left(-t \sqrt{\frac{\lambda}{3}}\right), a>0$ \\
\hline$\lambda>0$ & $\lambda_{F}=0$ & $b(t)=a \exp \left(\varepsilon t \sqrt{\frac{\lambda}{3}}\right), a>0, \varepsilon= \pm 1$ \\
\hline$\lambda>0$ & $\lambda_{F}<0$ & $b(t)=a \exp \left(t \sqrt{\frac{\lambda}{3}}\right)+\frac{3}{8} \frac{\lambda_{F}}{a \lambda} \exp \left(-t \sqrt{\frac{\lambda}{3}}\right), a \neq 0$ \\
\hline$\lambda=0$ & $\lambda_{F}=0$ & $b(t)=a, a>0$ \\
\hline$\lambda=0$ & $\lambda_{F}<0$ & $b(t)=\varepsilon \sqrt{\frac{-\lambda_{F}}{2}} t+a, \varepsilon= \pm 1$ \\
\hline$\lambda<0$ & $\lambda_{F}<0$ & $b(t)=a_{1} \cos \left(t \sqrt{-\frac{\lambda}{3}}\right)+a_{2} \operatorname{sen}\left(t \sqrt{-\frac{\lambda}{3}}\right), a_{1}^{2}+a_{2}^{2}=\frac{3}{2} \frac{\lambda_{F}}{\lambda}$ \\
\hline
\end{tabular}

onde $a, a_{1}$ e $a_{2} \in \mathbb{R}$ e $t_{1}, t_{2}$ tais que $I=\left(t_{1}, t_{2}\right)$ devem ser escolhidos de modo que $b(t)>0, \forall t \in I$.

O problema de encontrar um espaço-tempo generalizado de Kasner do tipo I que seja uma variedade de Einstein equivale a considerar $b=\varphi^{p}$ na discussão acima, onde $\varphi \in \mathcal{F}(I)$ e $p \in \mathbb{R}$. 


\subsection{Espaços-tempo de Kasner do tipo II}

Nesta seção busca-se uma classificação dos espaços-tempo de Kasner generalizados do tipo II que sejam variedades de Einstein ou possuam curvatura escalar constante.

É conveniente introduzir uma notação especial para certos sistemas de equações diferenciais.

Seja $\varphi \in \mathcal{F}(I)$ tal que $\left(\varphi^{\sigma}\right)^{\prime \prime}=\nu \varphi^{\sigma}$ e $\varphi(t)>0, \forall t \in I$, onde $\sigma, \nu \in \mathbb{R}$. Neste caso, será dito que $\varphi$ satisfaz $\left(\varphi^{\sigma} ; \nu\right)$. Todas as soluções de $\left(\varphi^{\sigma} ; \nu\right)$ são dadas por

$$
\varphi^{\sigma}(t)= \begin{cases}A e^{i t \sqrt{-\nu}}+B e^{-i t \sqrt{-\nu}}, & \text { se } \nu<0, \\ A t+B, & \text { se } \nu=0, \\ A e^{t \sqrt{\nu}}+B e^{-t \sqrt{\nu}}, & \text { se } \nu>0,\end{cases}
$$

onde as constantes $A$ e $B \in \mathbb{R}$ são tais que $\varphi(t)>0, \forall t \in I$.

Seja $\varphi \in \mathcal{F}(I)$ tal que

$$
\left(\varphi^{\sigma}\right)^{\prime \prime}=\nu \varphi^{\sigma},\left[\left(\varphi^{\sigma}\right)^{\prime}\right]^{2}=\nu\left(\varphi^{\sigma}\right)^{2} \text { e } \varphi(t)>0, \forall t \in I
$$

onde $\sigma, \nu \in \mathbb{R}$. Neste caso, será dito que $\varphi$ satisfaz $\left(\varphi^{\sigma} ; \nu ; *\right)$.

Note que, necessariamente, $\nu \geq 0$. Todas as soluções de $\left(\varphi^{\sigma} ; \nu ; *\right)$ são dadas por

$$
\varphi^{\sigma}(t)=A e^{ \pm t \sqrt{\nu}} \text {, onde } A \in \mathbb{R}, A>0 .
$$

Proposição 3.9 Se um espaço-tempo de Kasner generalizado do tipo $I I, M=$ $I \times_{\varphi^{p_{1}}} F_{1} \times_{\varphi^{p_{2}}} F_{2}$, é uma variedade de Einstein, então,

$$
\begin{aligned}
& (\eta-\zeta)\left(\frac{\varphi^{\prime}}{\varphi}\right)^{2}+\zeta \frac{\varphi^{\prime \prime}}{\varphi}=\lambda, \\
& p_{1}\left[(\zeta-1)\left(\frac{\varphi^{\prime}}{\varphi}\right)^{2}+\frac{\varphi^{\prime \prime}}{\varphi}\right]=\lambda e \\
& \frac{\lambda_{F_{2}}}{\varphi^{2 p_{2}}}+p_{2}\left[(\zeta-1)\left(\frac{\varphi^{\prime}}{\varphi}\right)^{2}+\frac{\varphi^{\prime \prime}}{\varphi}\right]=\lambda,
\end{aligned}
$$

onde $\zeta=p_{1}+2 p_{2}$ e $\eta=p_{1}^{2}+2 p_{2}^{2}$. 
Demonstração: É uma consequiência da Definição 3.2 e da Proposição 3.3.

A idéia, agora, é verificar no que resultam as equações da Proposição 3.9, quando os parâmetros $\eta, \zeta, \lambda$ e $\lambda_{F_{2}}$ assumem valores específicos.

Note que, de acordo com a Proposição $2.3, \lambda$ é constante, pois $M$ é suposta conexa. Portanto, utilizando o mesmo método de separação de variáveis da Proposição 2.12 , resulta da equação (3.15) que $\lambda_{F_{2}}$ é constante.

Lembrando que a métrica para estas variedades é dada por $-d t \otimes d t+\varphi^{2 p_{1}}(t) g_{F_{1}}+$ $\varphi^{2 p_{2}}(t) g_{F_{2}}$, obtém-se as seguintes possibilidades:

(a) $\zeta=0$.

Isto implica que $p_{2}=-\frac{1}{2} p_{1}$ e $\eta=\frac{3}{2} p_{1}^{2}$.

(a1) $\zeta=0$ e $\eta=0$.

Logo, $p_{1}=p_{2}=0$.

Da equação (3.14), $\lambda=0$ e da equação (3.15), $\lambda_{F_{2}}=0$.

Portanto, a métrica é dada por

$$
-d t \otimes d t+g_{F_{1}}+g_{F_{2}} .
$$

(a2) $\zeta=0$ e $\eta \neq 0$.

Então, $p_{1} \neq 0$ e $p_{2} \neq 0$.

(a2.1) $\zeta=0, \eta \neq 0$ e $\lambda_{F_{2}}=0$.

De $p_{2}=-\frac{1}{2} p_{1},(3.14)$ e (3.15), resulta que

$$
\frac{\lambda_{F_{2}}}{\varphi^{-p_{1}}}=\frac{3 \lambda}{2} \text {. }
$$

Portanto, como $\lambda_{F_{2}}=0$, segue que $\lambda=0$ e, de (3.13), $\varphi$ é constante, $\varphi(t)=\varphi_{0}, \forall t \in I, \varphi_{0} \in \mathbb{R}, \varphi_{0}>0$.

Portanto, a métrica é dada por

$$
-d t \otimes d t+\varphi_{0}^{2 p_{1}} g_{F_{1}}+\varphi_{0}^{2 p_{2}} g_{F_{2}} .
$$


(a2.2) $\zeta=0, \eta \neq 0$ e $\lambda_{F_{2}} \neq 0$.

De $p_{2}=-\frac{1}{2} p_{1},(3.14)$ e (3.15), resulta que

$$
\frac{\lambda_{F_{2}}}{\varphi^{-p_{1}}}=\frac{3 \lambda}{2} \text {. }
$$

Logo, $\lambda \neq 0$ e $\varphi$ é uma constante $\varphi_{0}$. Mas, isto não é compatível com a equação (3.13) e, portanto, neste caso, $M$ não pode ser uma variedade de Einstein.

(b) $\zeta \neq 0$.

Isto implica que $\eta \neq 0$. Neste caso, de acordo com a Proposição 3.4, as equações (3.13), (3.14) e (3.15) são equivalentes a

$$
\begin{aligned}
& \frac{\zeta^{2}}{\eta} \frac{\left(\varphi^{\eta / \zeta}\right)^{\prime \prime}}{\varphi^{\eta / \zeta}}=\lambda, \\
& \frac{p_{1}}{\zeta} \frac{\left(\varphi^{\zeta}\right)^{\prime \prime}}{\varphi^{\zeta}}=\lambda \quad \mathrm{e} \\
& \frac{\lambda_{F_{2}}}{\varphi^{2 p_{2}}}+\frac{p_{2}}{\zeta} \frac{\left(\varphi^{\zeta}\right)^{\prime \prime}}{\varphi^{\zeta}}=\lambda .
\end{aligned}
$$

(b1) $\zeta \neq 0$ e $\eta=\zeta^{2}$.

Note que $\zeta \neq 0$ implica que $p_{1} \neq 0$ ou $p_{2} \neq 0$.

Suponha $p_{1}=0$. Neste caso, $\eta=2 p_{2}^{2}$ e $\zeta^{2}=4 p_{2}^{2}$.

Portanto, $\eta \neq \zeta^{2}$. Logo, $p_{1} \neq 0$. De $\eta=\zeta^{2}, p_{1}^{2}+2 p_{2}^{2}=p_{1}^{2}+4 p_{2}^{2}+4 p_{1} p_{2}$, resulta que $p_{2}=0$ ou $p_{2}=-2 p_{1}$.

(b1.1) $\zeta \neq 0, \eta=\zeta^{2}$ e $\lambda=0$.

Como $p_{1} \neq 0$, das equações (3.17) e (3.18), resulta que $\lambda_{F_{2}}=0$.

Portanto, a métrica é dada por

$$
-d t \otimes d t+\varphi^{2 p_{1}} g_{F_{1}}+\varphi^{2 p_{2}} g_{F_{2}},
$$

onde $\varphi$ satisfaz $\left(\varphi^{\zeta} ; 0\right)$. 
(b1.2) $\zeta \neq 0, \eta=\zeta^{2}$ e $\lambda \neq 0$.

De (3.16) e (3.17), resulta que $p_{1}=\zeta$. Logo, $p_{2}=0$.

Portanto, de (3.18), $\lambda=\lambda_{F_{2}}$. Logo, a métrica é dada por

$$
-d t \otimes d t+\varphi^{2 \zeta} g_{F_{1}}+g_{F_{2}},
$$

onde $\varphi$ satisfaz $\left(\varphi^{\zeta} ; \lambda\right)$.

(b2) $\zeta \neq 0$ e $\eta \neq \zeta^{2}$.

Como $\eta \neq \zeta^{2}, p_{2} \neq 0$. De (3.17) e (3.18), resulta que

$$
\frac{p_{1}}{p_{2}} \frac{\lambda_{F_{2}}}{\varphi^{2 p_{2}}}=\left(\frac{p_{1}}{p_{2}}-1\right) \lambda .
$$

(b2.1) $\zeta \neq 0, \eta \neq \zeta^{2}$ e $\lambda=0$.

De (3.16), $\varphi^{\zeta}=(A t+B)^{\zeta^{2} / \eta}$, onde $A, B \in \mathbb{R}$ e

$$
\left(\varphi^{\zeta}\right)^{\prime \prime}=\frac{\zeta^{2}}{\eta}\left(\frac{\zeta^{2}}{\eta}-1\right)(A t+B)^{\left(\zeta^{2} / \eta\right)-2} A^{2} .
$$

(b2.1.1) $\zeta \neq 0, \eta \neq \zeta^{2}, \lambda=0$ e $\lambda_{F_{2}}=0$.

De (3.18), $\left(\varphi^{\zeta}\right)^{\prime \prime}(t)=0, \forall t \in I$. Logo, $A=0$. Portanto, $\varphi^{\zeta}$ é constante e $\varphi$ é uma constante positiva $\varphi_{0}$. Ou seja, a métrica é dada por

$$
-d t \otimes d t+\varphi_{0}^{2 p_{1}} g_{F_{1}}+\varphi_{0}^{2 p_{2}} g_{F_{2}} .
$$

(b2.1.2) $\zeta \neq 0, \eta \neq \zeta^{2}, \lambda=0$ e $\lambda_{F_{2}} \neq 0$.

De $\frac{p_{1}}{p_{2}} \frac{\lambda_{F_{2}}}{\varphi^{2 p_{2}}}=\left(\frac{p_{1}}{p_{2}}-1\right) \lambda$ resulta que $p_{1}=0$.

Portanto, $p_{2}=\frac{\zeta}{2}$ e $\frac{\eta}{\zeta^{2}}=\frac{1}{2}$. Logo, $\left(\varphi^{\zeta}\right)^{\prime \prime}=2 A^{2}$.

De (3.18), $\lambda_{F_{2}}=-A^{2}<0$. Ou seja, a métrica é dada por

$$
-d t \otimes d t+g_{F_{1}}+\varphi^{2 p_{2}} g_{F_{2}},
$$

onde $\varphi^{\zeta}=(A t+B)^{2}$. 
(b2.2) $\zeta \neq 0, \eta \neq \zeta^{2}$ e $\lambda \neq 0$.

De (3.17), $p_{1} \neq 0$. Logo,

$$
\frac{\lambda_{F_{2}}}{\varphi^{2 p_{2}}}=\left(1-\frac{p_{2}}{p_{1}}\right) \lambda
$$

(b2.2.1) $\zeta \neq 0, \eta \neq \zeta^{2}, \lambda \neq 0$ e $\lambda_{F_{2}}=0$.

Então, $p_{1}=p_{2}, \zeta=3 p_{1}, \eta=3 p_{1}^{2}$ e (3.16), (3.17) e (3.18) resultam em

$$
\frac{3\left(\varphi^{\zeta / 3}\right)^{\prime \prime}}{\varphi^{\zeta / 3}}=\frac{1}{3} \frac{\left(\varphi^{\zeta}\right)^{\prime \prime}}{\varphi^{\zeta}}=\lambda .
$$

Desenvolvendo as derivadas de segunda ordem, verifica-se que as equações acima implicam que $\left(\frac{\varphi^{\prime}}{\varphi}\right)^{2}=\frac{3 \lambda}{\zeta^{2}}$ e, portanto, $\left(\frac{\left(\varphi^{\zeta}\right)^{\prime}}{\varphi^{\zeta}}\right)^{2}=$ $3 \lambda$. Alternativamente, $\left(\frac{\left(\varphi^{\zeta}\right)^{\prime}}{\varphi^{\zeta}}\right)^{2}=3 \lambda$ implica que $\left(\frac{\varphi^{\prime}}{\varphi}\right)^{2}=\frac{3 \lambda}{\zeta^{2}}$. Desta última igualdade, resulta que $\left(\frac{\varphi^{\prime \prime}}{\varphi}\right)=\frac{3 \lambda}{\zeta^{2}}$, o que implica que $\frac{3\left(\varphi^{\zeta / 3}\right)^{\prime \prime}}{\varphi^{\zeta / 3}}=\frac{1}{3} \frac{\left(\varphi^{\zeta}\right)^{\prime \prime}}{\varphi^{\zeta}}=\lambda$.

Portanto, neste caso, a métrica é dada por

$$
-d t \otimes d t+\varphi^{2 p_{1}} g_{F_{1}}+\varphi^{2 p_{2}} g_{F_{2}}
$$

onde $\varphi$ satisfaz $\left(\varphi^{\zeta} ; 3 \lambda ; *\right)$.

(b2.2.2) $\zeta \neq 0, \eta \neq \zeta^{2}, \lambda \neq 0$ e $\lambda_{F_{2}} \neq 0$.

$\operatorname{De}\left(\frac{\lambda_{F_{2}}}{\varphi^{2 p_{2}}}\right)=\left(1-\frac{p_{2}}{p_{1}}\right) \lambda, 1-\frac{p_{2}}{p_{1}} \neq 0$. Como $\lambda$ e $\lambda_{F_{2}}$ são constantes, $\varphi$ é constante. Como $\zeta \neq 0$ implica em $\eta \neq 0$, por (3.16), isto resulta em uma contradição.

Logo, sob tais circunstâncias, não existe $M$ que seja variedade de Einstein.

Considera-se, agora, a classificação dos espaços-tempo de Kasner generalizados do tipo II com curvatura escalar constante. Neste caso, $\zeta=p_{1}+2 p_{2}$ e $\eta=p_{1}^{2}+2 p_{2}^{2}$. 
Serão utilizadas as Proposições 3.5 e 3.6, onde $S_{F_{1}}=0$, pois $F_{1}$ é unidimensional. Note que, se $S$ é constante, então, de acordo com a Proposição $2.12, S_{F_{2}}$ é constante.

(a1) $\zeta=0$ e $\eta=0$.

Então, $p_{1}=p_{2}=0$ e pela Proposição $3.5, S=S_{F_{2}}$.

A métrica é dada por

$$
-d t \otimes d t+g_{F_{1}}+g_{F_{2}} .
$$

(a2) $\zeta=0$ e $\eta \neq 0$.

Então, $p_{2}=-\frac{p_{1}}{2}$ e $\eta=6 p_{2}^{2}$.

A métrica é dada por

$$
-d t \otimes d t+\varphi^{2 p_{1}} g_{F_{1}}+\varphi^{2 p_{2}} g_{F_{2}}
$$

onde $\varphi$ satisfaz, pela Proposição 3.5,

$$
S=\eta\left(\frac{\varphi^{\prime}}{\varphi}\right)^{2}+\frac{S_{F_{2}}}{\varphi^{2 p_{2}}}
$$

(b) $\zeta \neq 0$.

Neste caso, $\eta \neq 0$ e pela Proposição 3.6,

$$
\frac{4 \mu^{\prime \prime}}{1+\frac{\eta}{\zeta^{2}}}=\mu S-S_{F_{2}} \mu^{\left(1-\left(4 /\left(1+\frac{\eta}{\zeta^{2}}\right)\right)\left(p_{2} / \zeta\right)\right)}
$$

onde $\mu=\varphi^{\left(\zeta^{2}+\eta\right) /(2 \zeta)}$. Em todos os casos particulares a seguir, a métrica é dada por $-d t \otimes d t+\varphi^{2 p_{1}} g_{F_{1}}+\varphi^{2 p_{2}} g_{F_{2}}$, onde as únicas soluções relevantes das respectivas equações diferenciais para a variável $\mu=\varphi^{\left(\zeta^{2}+\eta\right) /(2 \zeta)}$ serão aquelas que corresponderem a $\varphi(t)>0, \forall t \in I$.

(b1) $\zeta \neq 0$ e $\eta=\zeta^{2}$.

Então, $p_{1} \neq 0$ e $p_{2}=0$ ou $p_{2}=-2 p_{1}$. 
(b1.1) $\zeta \neq 0, \eta=\zeta^{2}$ e $S_{F_{2}}=0$.

Então, $2 \mu^{\prime \prime}=\mu S$.

(b1.2) $\zeta \neq 0, \eta=\zeta^{2}, S_{F_{2}} \neq 0$ e $p_{2}=0$.

Então, $2 \mu^{\prime \prime}=\mu\left(S-S_{F_{2}}\right)$.

(b1.3) $\zeta \neq 0, \eta=\zeta^{2}, S_{F_{2}} \neq 0$ e $p_{2}=-2 p_{1}$.

Então, $2 \mu^{\prime \prime}=\mu S-S_{F_{2}} \mu^{-1 / 3}$.

(b2) $\zeta \neq 0$ e $\eta \neq \zeta^{2}$.

Então, $p_{2} \neq 0$. Note que

$$
\begin{aligned}
& 2\left(p_{1}-p_{2}\right)^{2} \geq 0 \Rightarrow 2 p_{1}^{2}+2 p_{2}^{2} \geq 4 p_{1} p_{2} \\
& \Rightarrow 3 p_{1}^{2}+6 p_{2}^{2} \geq p_{1}^{2}+4 p_{2}^{2}+4 p_{1} p_{2} \Rightarrow 3 \eta \geq \zeta^{2} \Rightarrow \frac{\eta}{\zeta^{2}} \geq \frac{1}{3}
\end{aligned}
$$

(b2.1) $\zeta \neq 0, \eta \neq \zeta^{2}$ e $S_{F_{2}}=0$.

Então, $\frac{4 \mu^{\prime \prime}}{1+\left(\eta / \zeta^{2}\right)}=\mu S$.

(b2.2) $\zeta \neq 0, \eta \neq \zeta^{2}$ e $S_{F_{2}} \neq 0$.

Então, $\frac{4 \mu^{\prime \prime}}{1+\left(\eta / \zeta^{2}\right)}=\mu S-S_{F_{2}} \mu^{\left(1-\left(4 /\left(1+\frac{\eta}{\zeta^{2}}\right)\right)\left(p_{2} / \zeta\right)\right)}$.

Um caso particular corresponde a $\frac{\eta}{\zeta^{2}}=\frac{1}{3}$. Neste caso, $p_{1}=p_{2}=\frac{\zeta}{3} \mathrm{e}$ $3 \mu^{\prime \prime}=\mu S-S_{F_{2}}$.

\subsection{Espaços-tempo de Kasner do tipo III}

Nesta seção, busca-se uma classificação dos espaços-tempo de Kasner generalizados do tipo III que sejam variedades de Einstein ou possuam curvatura escalar constante. Neste caso, $\zeta=p_{1}+p_{2}+p_{3}$ e $\eta=p_{1}^{2}+p_{2}^{2}+p_{3}^{2}$.

Proposição 3.10 Se um espaço-tempo de Kasner generalizado $M=I \times \times_{\varphi^{p_{1}}} F_{1} \times_{\varphi^{p_{2}}}$ 
$F_{2} \times_{\varphi^{p_{3}}} F_{3}$ é uma variedade de Einstein, então,

$$
\begin{aligned}
& (\eta-\zeta)\left(\frac{\varphi^{\prime}}{\varphi}\right)^{2}+\zeta \frac{\varphi^{\prime \prime}}{\varphi}=\lambda, \\
& p_{1}\left[(\zeta-1)\left(\frac{\varphi^{\prime}}{\varphi}\right)^{2}+\frac{\varphi^{\prime \prime}}{\varphi}\right]=\lambda, \\
& p_{2}\left[(\zeta-1)\left(\frac{\varphi^{\prime}}{\varphi}\right)^{2}+\frac{\varphi^{\prime \prime}}{\varphi}\right]=\lambda \quad e \\
& p_{3}\left[(\zeta-1)\left(\frac{\varphi^{\prime}}{\varphi}\right)^{2}+\frac{\varphi^{\prime \prime}}{\varphi}\right]=\lambda .
\end{aligned}
$$

Demonstração: É uma conseqüência da Proposição 3.3 .

Note que, de acordo com a Proposição $2.3, \lambda$ é constante, pois $M$ é suposta conexa.

Somando as equações (3.20), (3.21) e (3.22) da proposição anterior, resulta que

$$
\zeta\left[(\zeta-1)\left(\frac{\varphi^{\prime}}{\varphi}\right)^{2}+\frac{\varphi^{\prime \prime}}{\varphi}\right]=3 \lambda .
$$

Considerando casos específicos:

(a) $\zeta=0$.

Então, de (3.23), $\lambda=0$.

(a1) $\zeta=0$ e $\eta=0$.

Logo, $p_{i}=0, \forall i \in\{1,2,3\}$. Portanto, a métrica é dada por

$$
-d t \otimes d t+g_{F_{1}}+g_{F_{2}}+g_{F_{3}} .
$$

(a2) $\zeta=0$ e $\eta \neq 0$.

Então, de (3.19), $\varphi$ é uma constante $\varphi_{0}$ e a métrica é dada por

$$
-d t \otimes d t+\varphi_{0}^{2 p_{1}} g_{F_{1}}+\varphi_{0}^{2 p_{2}} g_{F_{2}}+\varphi_{0}^{2 p_{3}} g_{F_{3}}
$$


(b) $\zeta \neq 0$.

Neste caso, de acordo com a Proposição 3.4, as equações de (3.19) a (3.22) são equivalentes a

$$
\begin{aligned}
& \frac{\zeta^{2}}{\eta} \frac{\left(\varphi^{\eta / \zeta}\right)^{\prime \prime}}{\varphi^{\eta / \zeta}}=\lambda, \\
& \frac{p_{1}}{\zeta} \frac{\left(\varphi^{\zeta}\right)^{\prime \prime}}{\varphi^{\zeta}}=\lambda, \\
& \frac{p_{2}}{\zeta} \frac{\left(\varphi^{\zeta}\right)^{\prime \prime}}{\varphi^{\zeta}}=\lambda \quad \mathrm{e} \\
& \frac{p_{3}}{\zeta} \frac{\left(\varphi^{\zeta}\right)^{\prime \prime}}{\varphi^{\zeta}}=\lambda .
\end{aligned}
$$

Somando as três últimas equações,

$$
\frac{\left(\varphi^{\zeta}\right)^{\prime \prime}}{\varphi^{\zeta}}=3 \lambda
$$

(b1) $\zeta \neq 0$ e $\eta=\zeta^{2}$.

Neste caso, comparando (3.24) e (3.28), resulta que $\lambda=3 \lambda$, logo, $\lambda=0$.

Portanto, a métrica é dada por

$$
-d t \otimes d t+\varphi^{2 p_{2}} g_{F_{1}}+\varphi^{2 p_{2}} g_{F_{2}}+\varphi^{2 p_{3}} g_{F_{3}}
$$

onde $\varphi$ satisfaz $\left(\varphi^{\zeta} ; 0\right)$, conforme a notação da seção anterior.

(b2) $\zeta \neq 0$ e $\eta \neq \zeta^{2}$.

Como $\eta=p_{1}^{2}+p_{2}^{2}+p_{3}^{2}$ e $\zeta^{2}=\eta+2\left(p_{1} p_{2}+p_{1} p_{3}+p_{2} p_{3}\right), \eta \neq \zeta^{2}$ implica que ao menos dois dos parâmetros entre $p_{1}, p_{2}$ e $p_{3}$ são diferentes de zero.

(b2.1) $\zeta \neq 0, \eta \neq \zeta^{2}$ e $\lambda=0$.

Então, de (3.24), $\varphi^{\zeta}=(A t+B)^{\zeta^{2} / \eta}$, onde $A, B \in \mathbb{R}$ e

$$
\left(\varphi^{\zeta}\right)^{\prime \prime}=\frac{\zeta^{2}}{\eta}\left(\frac{\zeta^{2}}{\eta}-1\right)(A t+B)^{\left(\zeta^{2} / \eta\right)-2} A^{2}
$$


Por (3.28), $A=0$. Portanto, $\varphi$ é uma constante positiva $\varphi_{0}$ e a métrica é dada por

$$
-d t \otimes d t+\varphi_{0}^{2 p_{1}} g_{F_{1}}+\varphi_{0}^{2 p_{2}} g_{F_{2}}+\varphi_{0}^{2 p_{3}} g_{F_{3}}
$$

(b2.2) $\zeta \neq 0, \eta \neq \zeta^{2}$ e $\lambda \neq 0$.

Como ao menos dois parâmetros entre $p_{1}, p_{2}$ e $p_{3}$ são diferentes de zero, escolha uma equação entre (3.25), (3.26) e (3.27), tal que o valor de $p_{i}$ associado seja não nulo. Resulta que $\left(\varphi^{\zeta}\right)^{\prime \prime} \neq 0$. Logo, de (3.25), (3.26) , (3.27) e (3.28),

$$
p_{1}=p_{2}=p_{3}=\frac{\lambda \zeta \varphi^{\zeta}}{\left(\varphi^{\zeta}\right)^{\prime \prime}}=\frac{\zeta}{3}
$$

Portanto, $\eta=3 p_{1}^{2}, \zeta^{2}=9 p_{1}^{2}$ e $\frac{\eta}{\zeta^{2}}=\frac{1}{3}$.

De (3.24) a (3.27), resulta que

$$
\frac{3\left(\varphi^{\zeta / 3}\right)^{\prime \prime}}{\varphi^{\zeta / 3}}=\frac{1}{3} \frac{\left(\varphi^{\zeta}\right)^{\prime \prime}}{\varphi^{\zeta}}=\lambda
$$

Conforme discutido na seção anterior, isto equivale a dizer que $\varphi$ satisfaz $\left(\varphi^{\zeta} ; 3 \lambda ; *\right) \operatorname{com} \lambda>0$, pois $\left[\left(\varphi^{\zeta}\right)^{\prime}\right]^{2}=3 \lambda\left(\varphi^{\zeta}\right)^{2}$.

Note que, como $S_{F_{1}}=S_{F_{2}}=S_{F_{3}}=0$, pois $\operatorname{dim}\left(F_{1}\right)=\operatorname{dim}\left(F_{2}\right)=\operatorname{dim}\left(F_{3}\right)=1$, a discussão a respeito dos espaços-tempo de Kasner generalizados do tipo III de curvatura escalar constante está contida no Exemplo 3.7, pois o mesmo inclui todos os valores possíveis dos parâmetros $\eta$ e $\zeta$. 



\section{Capítulo 4}

\section{Espaços-tempo BTZ generalizados}

Os principais resultados deste capítulo estão contidos na referência [13].

Antes de apresentar os espaços-tempo BTZ (Bañados, Teitelboim e Zanelli) generalizados, cabem, aqui, algumas palavras a respeito do espaço-tempo BTZ usual ou clássico estático. Para maiores detalhes, veja [3].

A métrica para o espaço-tempo BTZ estático na parte interior do horizonte de eventos do buraco negro é dada por

$$
d s^{2}=N^{2} d t^{2}-N^{-2} d r^{2}+r^{2} d \phi^{2}
$$

onde $N^{2}=m-\frac{r^{2}}{l^{2}},-\infty<t<\infty, 0<r<m^{1 / 2} l, 0 \leq \phi \leq 2 \pi, m$ e $l \in \mathbb{R}$ são constantes positivas.

Em [16], observa-se que tal métrica pode ser colocada na forma da métrica de um espaço-tempo de Robertson-Walker generalizado, através de uma mudança de coordenadas convenientemente escolhida, na forma a seguir. Defina uma nova coordenada $\mu$ por $d \mu^{2}=N^{-2} d r^{2}$. Integrando, isto resulta em

$$
F(r):=\mu=\int_{0}^{r} \frac{\mathrm{d} y}{N(y)}
$$

onde a constante de integração é tomada igual a zero, ou seja,

$$
\mu=l \operatorname{arcsen}\left(\frac{r}{m^{1 / 2} l}\right)
$$


com as condições de contorno

$$
\begin{aligned}
& F(r) \rightarrow \frac{l \pi}{2}, \text { para } r \rightarrow m^{1 / 2} l \text { e } \\
& F(r) \rightarrow 0, \text { para } r \rightarrow 0 .
\end{aligned}
$$

Logo, $r=F^{-1}(\mu)=m^{1 / 2} l \operatorname{sen}\left(\frac{\mu}{l}\right)$.

Portanto,

$$
d s^{2}=-d \mu^{2}+b_{1}^{2}(\mu) d t^{2}+b_{2}^{2}(\mu) d \phi^{2},
$$

onde $b_{1}(\mu)=\left(m-\frac{\left(F^{-1}(\mu)\right)^{2}}{l^{2}}\right)^{1 / 2}, b_{2}(\mu)=F^{-1}(\mu)$. Isto é,

$$
d s^{2}=-d \mu^{2}+m \cos ^{2}\left(\frac{\mu}{l}\right) d t^{2}+m l^{2} \operatorname{sen}^{2}\left(\frac{\mu}{l}\right) d \phi^{2} .
$$

Definição 4.1 Um espaço-tempo BTZ generalizado é definido do seguinte modo: Considere a métrica

$$
d s^{2}=-N^{-2} d r^{2}+N^{2} d t^{2}+r^{2} d \phi^{2}
$$

onde $r \in\left(0, r_{1}\right), t \in T, \phi \in U, T$ e $U$ intervalos (conexos) em $\mathbb{R}, 0<r_{1}<\infty$ e $N \in \mathcal{F}\left(\left(0, r_{1}\right)\right)$. Suponha que a função $F$ definida por

$$
F(r):=\mu=\int_{0}^{r} \frac{\mathrm{d} y}{N(y)}+a_{1}
$$

onde $a_{1} \in \mathbb{R}$ é uma constante arbitrária, é um difeomorfismo sobre sua imagem. Então, $r=F^{-1}(\mu) \mathrm{e}$

$$
d s^{2}=-d \mu^{2}+b_{1}^{2}(\mu) d t^{2}+b_{2}^{2}(\mu) d \phi^{2}
$$

onde $b_{1}(\mu)=N\left(F^{-1}(\mu)\right)$ e $b_{2}(\mu)=F^{-1}(\mu)$.

Esta construção é um caso particular de um espaço-tempo de Robertson-Walker generalizado. 
Note que

$$
\begin{aligned}
\frac{\mathrm{d}}{\mathrm{d} r} F(r) & =\frac{1}{N(r)} \mathrm{e} \\
1 & =\frac{\mathrm{d}}{\mathrm{d} \mu}\left(F \circ F^{-1}\right)(\mu)=\left(\left.\frac{\mathrm{d}}{\mathrm{d} r} F(r)\right|_{r=F^{-1}(\mu)}\right) \frac{\mathrm{d}}{\mathrm{d} \mu} F^{-1}(\mu) .
\end{aligned}
$$

Portanto,

$$
b_{1}(\mu)=N\left(b_{2}(\mu)\right)
$$

e, utilizando (4.1) e (4.2),

$$
\frac{\mathrm{d} b_{2}(\mu)}{\mathrm{d} \mu}=\frac{\mathrm{d}}{\mathrm{d} \mu} F^{-1}(\mu)=N\left(F^{-1}(\mu)\right)=b_{1}(\mu) .
$$

Por (4.3) e (4.4),

$$
\frac{\mathrm{d} b_{2}(\mu)}{\mathrm{d} \mu}=N\left(b_{2}(\mu)\right) .
$$

Utilizando, agora, (4.4) e (4.5),

$\frac{\mathrm{d} b_{1}(\mu)}{\mathrm{d} \mu}=\frac{\mathrm{d}^{2} b_{2}(\mu)}{\mathrm{d} \mu^{2}}=\left(\left.\frac{\mathrm{d}}{\mathrm{d} r} N(r)\right|_{r=b_{2}(\mu)}\right) \frac{\mathrm{d} b_{2}(\mu)}{\mathrm{d} \mu}=\left(\left.\frac{\mathrm{d}}{\mathrm{d} r} N(r)\right|_{r=b_{2}(\mu)}\right) b_{1}(\mu)$.

As relações (4.6) e (4.4) permitem obter

$$
\begin{aligned}
\frac{\mathrm{d}^{2} b_{1}(\mu)}{\mathrm{d} \mu^{2}} & =b_{1}(\mu)\left(\left.\frac{\mathrm{d}^{2} N(r)}{\mathrm{d} r^{2}}\right|_{r=b_{2}(\mu)}\right)\left(\frac{\mathrm{d}}{\mathrm{d} \mu} b_{2}(\mu)\right)+\left(\left.\frac{\mathrm{d}}{\mathrm{d} r} N(r)\right|_{r=b_{2}(\mu)}\right) \frac{\mathrm{d}}{\mathrm{d} \mu} b_{1}(\mu) \\
& =\left(\left.\frac{\mathrm{d}^{2} N(r)}{\mathrm{d} r^{2}}\right|_{r=b_{2}(\mu)}\right) b_{1}^{2}(\mu)+\left(\left.\frac{\mathrm{d}}{\mathrm{d} r} N(r)\right|_{r=b_{2}(\mu)}\right)^{2} b_{1}(\mu) .
\end{aligned}
$$

Por (4.7) e (4.3),

$$
\begin{aligned}
\frac{1}{b_{1}(\mu)} \frac{\mathrm{d}^{2} b_{1}(\mu)}{\mathrm{d} \mu^{2}} & =\left(\left.\frac{\mathrm{d}^{2} N(r)}{\mathrm{d} r^{2}}\right|_{r=b_{2}(\mu)}\right) b_{1}(\mu)+\left(\left.\frac{\mathrm{d}}{\mathrm{d} r} N(r)\right|_{r=b_{2}(\mu)}\right)^{2} \\
& =\left(\left.\frac{\mathrm{d}^{2} N(r)}{\mathrm{d} r^{2}}\right|_{r=b_{2}(\mu)}\right) N\left(b_{2}(\mu)\right)+\left(\left.\frac{\mathrm{d}}{\mathrm{d} r} N(r)\right|_{r=b_{2}(\mu)}\right)^{2} \\
& =\left.\frac{\mathrm{d}}{\mathrm{d} r}\left(\frac{\mathrm{d} N(r)}{\mathrm{d} r} N(r)\right)\right|_{r=b_{2}(\mu)}=\left.\frac{1}{2} \frac{\mathrm{d}^{2}}{\mathrm{~d} r^{2}} N^{2}(r)\right|_{r=b_{2}(\mu)} .
\end{aligned}
$$

Utilizando (4.6) e (4.3),

$$
\frac{1}{b_{2}(\mu)} \frac{\mathrm{d}^{2} b_{2}(\mu)}{\mathrm{d} \mu^{2}}=\left(\left.\frac{\mathrm{d}}{\mathrm{d} r} N(r)\right|_{r=b_{2}(\mu)}\right) \frac{N\left(b_{2}(\mu)\right)}{b_{2}(\mu)}=\left.\frac{1}{2 b_{2}(\mu)}\left(\frac{\mathrm{d}}{\mathrm{d} r} N^{2}(r)\right)\right|_{r=b_{2}(\mu)} .
$$


Por (4.4) e (4.6),

$$
\begin{aligned}
\frac{1}{b_{1}(\mu) b_{2}(\mu)} \frac{\mathrm{d}}{\mathrm{d} \mu} b_{1}(\mu) \frac{\mathrm{d}}{\mathrm{d} \mu} b_{2}(\mu) & =\left.\frac{b_{1}^{2}(\mu)}{b_{1}(\mu) b_{2}(\mu)} \frac{\mathrm{d}}{\mathrm{d} r} N(r)\right|_{r=b_{2}(\mu)} \\
& =\left.\frac{b_{1}(\mu)}{b_{2}(\mu)} \frac{\mathrm{d}}{\mathrm{d} r} N(r)\right|_{r=b_{2}(\mu)}=\frac{1}{b_{2}(\mu)} \frac{\mathrm{d}^{2} b_{2}(\mu)}{\mathrm{d} \mu^{2}} .
\end{aligned}
$$

Proposição 4.2 Considere um espaço-tempo BTZ generalizado, conforme a Definição 4.1. Então, tal variedade é uma variedade de Einstein se e somente se

$$
N^{2}(r)=\frac{\lambda r^{2}}{2}+C_{1}
$$

onde $\lambda \in \mathbb{R}$ é a curvatura de Ricci e $C_{1} \in \mathbb{R}$ é uma constante arbitrária.

Demonstração: De acordo com o Teorema 2.8, tal variedade é de Einstein se e somente se

$$
\begin{aligned}
& \frac{1}{b_{1}(\mu)} \frac{\mathrm{d}^{2} b_{1}(\mu)}{\mathrm{d} \mu^{2}}+\frac{1}{b_{2}(\mu)} \frac{\mathrm{d}^{2} b_{2}(\mu)}{\mathrm{d} \mu^{2}}=\lambda, \\
& \frac{1}{b_{1}(\mu)} \frac{\mathrm{d}^{2} b_{1}(\mu)}{\mathrm{d} \mu^{2}}+\frac{1}{b_{1}(\mu) b_{2}(\mu)} \frac{\mathrm{d} b_{1}(\mu)}{\mathrm{d} \mu} \frac{\mathrm{d} b_{2}(\mu)}{\mathrm{d} \mu}=\lambda \quad \text { e } \\
& \frac{1}{b_{2}(\mu)} \frac{\mathrm{d}^{2} b_{2}(\mu)}{\mathrm{d} \mu^{2}}+\frac{1}{b_{1}(\mu) b_{2}(\mu)} \frac{\mathrm{d} b_{1}(\mu)}{\mathrm{d} \mu} \frac{\mathrm{d} b_{2}(\mu)}{\mathrm{d} \mu}=\lambda .
\end{aligned}
$$

É possível reescrever estas equações, utilizando (4.8), (4.9) e (4.10):

$$
\begin{aligned}
& \frac{d^{2}\left(N^{2}(r)\right)}{\mathrm{d} r^{2}}+\frac{1}{r} \frac{\mathrm{d}}{\mathrm{d} r}\left(N^{2}(r)\right)=2 \lambda, \\
& \frac{\mathrm{d}^{2}\left(N^{2}(r)\right)}{\mathrm{d} r^{2}}+\frac{1}{r} \frac{\mathrm{d}}{\mathrm{d} r}\left(N^{2}(r)\right)=2 \lambda \quad \text { e } \\
& \frac{1}{r} \frac{\mathrm{d}}{\mathrm{d} r}\left(N^{2}(r)\right)=\lambda,
\end{aligned}
$$

onde as funções acima são avaliadas em $r=b_{2}(\mu)$. Como $\mu$ é arbitrário e as equações (4.14) e (4.15) são iguais, segue que é necessário e suficiente na demonstração da proposição considerar que, $\forall r \in\left(0, r_{1}\right)$, as equações (4.15) e (4.16) são satisfeitas.

De (4.15) e (4.16), resulta que

$$
\frac{\mathrm{d}^{2}\left(N^{2}(r)\right)}{\mathrm{d} r^{2}}=\lambda
$$


ou seja,

$$
N^{2}(r)=\frac{\lambda r^{2}}{2}+C_{2} r+C_{1}
$$

onde $C_{1}$ e $C_{2} \in \mathbb{R}$ são constantes arbitrárias. Utilizando (4.16), resulta que $C_{2}=0$, o que encerra a demonstração.

Observação: Note que o espaço-tempo BTZ estático clássico descrito no começo do capítulo é uma variedade de Einstein tal que $\lambda=\frac{-2}{l^{2}}$, o que é constante, pois $l$ é uma constante.

De acordo com a Proposição 2.11, equação (2.11), a curvatura escalar de um espaço-tempo BTZ generalizado é dada por

$$
\begin{aligned}
S & =2\left(\frac{b_{1}^{\prime \prime}(\mu)}{b_{1}(\mu)}+\frac{b_{2}^{\prime \prime}(\mu)}{b_{2}(\mu)}+\frac{b_{1}^{\prime}(\mu) b_{2}^{\prime}(\mu)}{b_{1}(\mu) b_{2}(\mu)}\right) \\
& =2\left(\frac{b_{1}^{\prime \prime}(\mu)}{b_{1}(\mu)}+\frac{2 b_{2}^{\prime \prime}(\mu)}{b_{2}(\mu)}\right) \\
& =\left.\frac{\mathrm{d}^{2}\left(N^{2}(r)\right)}{\mathrm{d} r^{2}}\right|_{r=b_{2}(\mu)}+\left.2\left(\frac{\mathrm{d}}{\mathrm{d} r} N^{2}(r)\right)\right|_{r=b_{2}(\mu)} \frac{1}{b_{2}(\mu)}
\end{aligned}
$$

conforme (4.8), (4.9) e (4.10).

Como $\mu$ é arbitrário,

$$
S=\frac{\mathrm{d}^{2}\left(N^{2}(r)\right)}{\mathrm{d} r^{2}}+\frac{2}{r} \frac{\mathrm{d}}{\mathrm{d} r}\left(N^{2}(r)\right)
$$

Proposição 4.3 Considere um espaço-tempo BTZ generalizado, conforme a Definição 4.1. Então, a curvatura escalar $S$ de tal variedade é constante, onde $S=\gamma$, se e somente se

$$
N^{2}(r)=-\frac{C_{1}}{r}+\frac{\gamma r^{2}}{6}+C_{2}
$$

onde $C_{1}, C_{2} \in \mathbb{R}$ são constantes arbitrárias e $\gamma \in \mathbb{R}$.

\section{Demonstração:}


(a) Suponha $N^{2}(r)=-\frac{C_{1}}{r}+\frac{\gamma r^{2}}{6}+C_{2}$, onde $C_{1}, C_{2}$ e $\gamma \in \mathbb{R}$ são constantes. Por (4.18),

$$
S=-\frac{2 C_{1}}{r^{3}}+\frac{\gamma}{3}+\frac{2}{r}\left(\frac{C_{1}}{r^{2}}+\frac{\gamma r}{3}\right)=\gamma
$$

portanto, $S$ é constante.

(b) Suponha $S$ constante. Então, a solução geral da equação diferencial (4.18), no caso em que $S$ é uma constante, é dada por

$$
N^{2}(r)=-\frac{C_{1}}{r}+\frac{r^{2} S}{6}+C_{2}
$$

onde $C_{1}$ e $C_{2} \in \mathbb{R}$ são constantes arbitrárias.

Observação: Note que a curvatura escalar do espaço-tempo BTZ estático clássico descrito no começo do capítulo é constante e dada por $S=-\frac{6}{l^{2}}$. Conforme visto neste capítulo, tal variedade é, também, uma variedade de Einstein, onde a curvatura de Ricci é dada por $\lambda=-\frac{2}{l^{2}}$. Note que $S=3 \lambda$. Conforme a Proposição 2.3, isto era previsível, pois a dimensão desta variedade é igual a três. 


\section{Apêndice}

Neste apêndice são apresentados alguns dos conceitos básicos da geometria semiRiemanniana, necessários ao desenvolvimento do trabalho. Além disso, são estabelecidas a nomenclatura e algumas convenções.

No que concerne a esta seção, todos os conceitos e resultados com as suas respectivas demonstrações podem ser encontrados no livro de O'Neill [22]. Entretanto, algumas das convenções utilizadas seguem o livro de Beem, Ehrlich e Easley [5].

O conjunto das funções de classe $C^{\infty}$ em uma variedade $M$ é denotado por $\mathcal{F}(M)$. Todas as variedades são supostas com estrutura diferenciável de classe $C^{\infty}$. Além disso, se necessário, consideram-se implícitas as propriedades de Hausdorff, paracompacticidade e conectividade.

Uma variedade semi-Riemanniana é uma variedade com tensor métrico arbitrário. Uma variedade é Riemanniana se sua métrica tem assinatura $(+,+, \ldots,+)$, enquanto que uma variedade de Lorentz corresponde ao caso $(-,+,+, \ldots,+)$.

Por $\mathscr{T}(M)$ entende-se o conjunto dos campos vetoriais de classe $C^{\infty}$ na variedade $M$.

Dada uma variedade produto $M \times N$, o espaço tangente no ponto $(p, q) \in M \times N$, $T_{(p, q)}(M \times N)$, é a soma direta de seus subespaços $T_{(p, q)} M \equiv T_{(p, q)}(M \times q)$ e $T_{(p, q)} N \equiv$ $T_{(p, q)}(p \times N), \forall p \in M$ e $\forall q \in N$. Para um vetor $v \in T_{(p, q)} M\left(T_{(p, q)} N\right)$, a nomenclatura "tangente à variedade $M(N)$ " será utilizada.

Para relacionar o cálculo em $M \times N$ ao cálculo em $M$ e $N$, o conceito utilizado 
é o de levantamento. As projeções canônicas são definidas por $\pi: M \times N \rightarrow M$, $\pi(p, q)=p$ e $\sigma: M \times N \rightarrow N, \sigma(p, q)=q$. Se $f \in \mathcal{F}(M)$, o levantamento de $f$ para $M \times N$ é definido por $\bar{f}=f \circ \pi \in \mathcal{F}(M \times N)$.

Se $v \in T_{p} M$ e $q \in N$, então, o levantamento $\bar{v}$ de $v$ para $(p, q)$ é o único vetor em $T_{(p, q)} M$ tal que $\mathrm{d} \pi(\bar{v})=v$. Se $X \in \Re(M)$, o levantamento de $X$ para $M \times N$ é o campo vetorial $\bar{X} \in \mathscr{X}(M \times N)$ cujo valor $\forall(p, q) \in M \times N$ é o levantamento de $X_{p}$ para $(p, q)$. O conjunto de todos os levantamentos é denotado por $\mathcal{L}(M) \subset \Re(M \times N)$. Levantamentos de $N$ são definidos de modo completamente análogo.

Proposição A.1 (1) Se $\bar{X}, \bar{Y} \in \mathcal{L}(M)$, então, $[\bar{X}, \bar{Y}] \in \mathcal{L}(M)$ é o levantamento de $[X, Y]$. Similarmente para $\mathcal{L}(N)$.

(2) $S e \bar{X} \in \mathcal{L}(M)$ e $\bar{V} \in \mathcal{L}(N)$, então, $[\bar{X}, \bar{V}]=0$.

Para um tensor covariante $A$ em $M$ de ordem $(0, s)$, o levantamento $\bar{A}$ é dado pelo "pull-back", $\pi^{*}(A)$. Para um tensor $A$ em $M$ de ordem $(1, s), A: \mathfrak{X}(M)^{s} \rightarrow$ $\dddot{X}(M)$, se $v_{1}, \ldots, v_{s} \in T_{(p, q)}(M \times N)$, defina $\bar{A}\left(v_{1}, \ldots, v_{s}\right)$ como o vetor tangente a $M$ em $(p, q)$, tal que $\mathrm{d} \pi\left(\bar{A}\left(v_{1}, \ldots, v_{s}\right)\right)=A\left(\mathrm{~d} \pi v_{1}, \ldots, \mathrm{d} \pi v_{s}\right)$. Definições análogas são utilizadas para os levantamentos de tensores de ordem $(0, s)$ ou $(1, s)$ em $N$.

O conceito de levantamento em uma variedade produto com um número arbitrário de fatores, $M_{1} \times \cdots \times M_{n}$, é completamente análogo ao caso $n=2$.

Seguem-se, agora, alguns resultados e definições válidos para qualquer variedade semi-Riemanniana $M$, onde $n=\operatorname{dim}(M)$.

Definição A.2 Um tensor métrico $g$ em uma variedade $M$ de classe $C^{\infty}$ é um campo tensorial não degenerado e simétrico, de ordem $(0,2)$, de índice constante. 
Proposição A.3 Em toda variedade $M$, dado um ponto $p \in M$ arbitrário, existe um conjunto $E_{1}, \ldots, E_{n}$ de campos vetoriais em uma vizinhança $U$ de $p$, satisfazendo as propriedades a seguir:

$$
\begin{aligned}
& g\left(E_{i}, E_{j}\right)_{q}=0, \text { se } i \neq j, \\
& g\left(E_{i}, E_{i}\right)_{q}=\varepsilon_{i}= \pm 1, \forall i, j \in\{1, \ldots, n\} e \\
& g(V, W)_{q}=\sum_{i=1}^{n} \varepsilon_{i} g\left(V, E_{i}\right)_{q} g\left(W, E_{i}\right)_{q},
\end{aligned}
$$

$\forall q \in U, \forall V, W \in \mathscr{X}(M) . \quad O$ conjunto $\left\{E_{i}\right\}$ satisfazendo as condições acima é denominado um referencial ortonormal móvel. A assinatura da métrica é dada por $\left(\varepsilon_{1}, \ldots, \varepsilon_{n}\right)$.

Definição A.4 Uma conexão $D$ em uma variedade $M$ de classe $C^{\infty}$ é uma aplicação $D: \mathscr{X}(M) \times \mathscr{X}(M) \rightarrow \mathscr{X}(M)$ tal que

(1) $D_{V} W$ é $\mathcal{F}(M)$-linear em $V$,

(2) $D_{V} W$ é $\mathbb{R}$-linear em $W$ e

(3) $D_{V}(f W)=(V f) W+f D_{V} W$, para todo $V, W \in \mathscr{X}(M)$, para todo $f \in \mathcal{F}(M)$.

$D_{V} W$ é a derivada covariante de $W$ com respeito a $V$ para a conexão $D$.

Teorema A.5 Em uma variedade $M$ existe uma única conexão $D$ tal que

(1) $[V, W]=D_{V} W-D_{W} V e$

(2) $X g(V, W)=g\left(D_{X} V, W\right)+g\left(V, D_{X} W\right)$,

para todo $X, V, W \in \mathscr{X}(M)$. D é denominado a conexão de Levi-Civita em $M$ e é caracterizado pela fórmula de Koszul

$$
\begin{aligned}
2 g\left(D_{V} W, X\right)= & V g(W, X)+W g(X, V)-X g(V, W) \\
& -g(V,[W, X])+g(W,[X, V])+g(X,[V, W]) .
\end{aligned}
$$


Seja $\left\{x_{i}\right\}$ um sistema de coordenadas definido localmente. Os símbolos de Christoffel são definidos por $D_{\frac{\partial}{\partial x^{i}}} \frac{\partial}{\partial x^{j}}=\sum_{k=1}^{n} \Gamma_{i j}^{k} \frac{\partial}{\partial x^{k}}$.

Proposição A.6 Em toda variedade $M$, dado um ponto $p \in M$ arbitrário, existe um referencial ortonormal móvel $E_{1}, \ldots, E_{n}$ em uma vizinhança $U$ de $p$, satisfazendo: $D_{E_{i}} E_{j}(p)=0$. O conjunto $\left\{E_{i}\right\}$ é denominado um referencial geodésico.

Proposição A.7 Seja M uma variedade semi-Riemanniana com conexão de LeviCivita D. Então, a aplicação $R:[\mathscr{X}(M)]^{3} \rightarrow \mathscr{X}(M)$ dada por

$$
R(X, Y) Z=\left[D_{X}, D_{Y}\right] Z-D_{[X, Y]} Z
$$

é um campo tensorial de ordem $(1,3)$ em $M$ denominado o tensor de curvatura de Riemann de $M$. Além disso, $\forall x, y, v, w \in T_{p} M, \forall p \in M$,

(1) $R(x, y) v=-R(y, x) v$,

(2) $g(R(x, y) v, w)=-g(R(x, y) w, v) e$

(3) $g(R(x, y) v, w)=g(R(v, w) x, y)$.

Dada uma variedade semi-Riemanniana arbitrária $M$, a segunda identidade de Bianchi é satisfeita:

$$
\left(D_{Z} R\right)(X, Y) V+\left(D_{X} R\right)(Y, Z) V+\left(D_{Y} R\right)(Z, X) V=0
$$

$\forall X, Y, Z$ e $V \in \mathscr{X}(M)$, onde

$\left(D_{Z} R\right)(X, Y) V \equiv D_{Z}(R(X, Y) V)-R\left(D_{Z} X, Y\right) V-R\left(X, D_{Z} Y\right) V-R(X, Y) D_{Z} V$

Dados um ponto $p \in M$ arbitrário e uma base arbitrária $\left\{E_{i}\right\}$ de $T_{p} M$, define-se

$$
R\left(E_{i}, E_{j}\right) E_{k}=\sum_{l=1}^{n} R_{k i j}^{l} E_{l}, \quad \forall i, j, k \in\{1, \ldots, n\} .
$$


Dado um sistema de coordenadas $\left\{x_{i}\right\}$ definido localmente,

$$
R_{j k l}^{i}=-\frac{\partial}{\partial x^{l}} \Gamma_{k j}^{i}+\frac{\partial}{\partial x^{k}} \Gamma_{l j}^{i}-\sum_{m=1}^{n} \Gamma_{l m}^{i} \Gamma_{k j}^{m}+\sum_{m=1}^{n} \Gamma_{k m}^{i} \Gamma_{l j}^{m} .
$$

Observação: As definições aqui utilizadas para o tensor de Riemann, o tensor de Ricci e a curvatura escalar seguem a referência [5].

Proposição A.8 Sejam $M$ uma variedade semi-Riemanniana com tensor de Riemann $R$ e $\left\{E_{i}\right\}$ uma base arbitrária de $T_{p} M$, onde $p \in M$ é arbitrário. Então, o tensor de Ricci é definido como a contração do tensor de Riemann cujos componentes em relação à base $\left\{E_{i}\right\}$ são dados por:

$$
\operatorname{Ric}\left(E_{i}, E_{j}\right)=\operatorname{Ric}_{i j}=\sum_{k=1}^{n} R_{i k j}^{k}, \quad \forall i, j \in\{1, \ldots, n\} .
$$

A aplicação Ric : $[\mathscr{X}(M)]^{2} \rightarrow \mathcal{F}(M)$ é um campo tensorial simétrico de ordem $(0,2)$. $S e\left\{E_{i}\right\}$ é um referencial ortonormal móvel, então, $\forall X, Y \in \mathscr{X}(M)$,

$$
\operatorname{Ric}(X, Y)=\sum_{i=1}^{n} g\left(E_{i}, E_{i}\right) g\left(R\left(X, E_{i}\right) E_{i}, Y\right) .
$$

Observação: Nas definições e resultados a seguir denota-se $g_{i j}=g\left(E_{i}, E_{j}\right)$, onde $\left\{E_{i}\right\}$ é uma base arbitrária de $T_{p} M$ e $p \in M$ é arbitrário. Além disso, $g^{i j}$ é a matriz inversa de $g_{i j}$.

Proposição A.9 Sejam $M$ uma variedade semi-Riemanniana e $\left\{E_{i}\right\}$ uma base arbitrária de $T_{p} M$, onde $p \in M$ é arbitrário. Então, a curvatura escalar é definida como a contração do tensor de Ricci que, em relação à base $\left\{E_{i}\right\}$, é dada por:

$$
S=\sum_{i, j=1}^{n} g^{i j} \operatorname{Ric}_{i j}
$$

Se $\left\{E_{i}\right\}$ é um referencial ortonormal móvel, então,

$$
S=\sum_{i=1}^{n} g\left(E_{i}, E_{i}\right) \operatorname{Ric}\left(E_{i}, E_{i}\right)
$$


Proposição A.10 O gradiente de uma função $f \in \mathcal{F}(M)$, definido por

$$
g(\operatorname{grad} f, X)=X(f), \forall X \in \Re(M),
$$

é um campo vetorial que, em termos de um sistema de coordenadas local, é dado por

$$
\operatorname{grad} f=\sum_{i, j=1}^{n} g^{i j} \frac{\partial f}{\partial x^{i}} \frac{\partial}{\partial x^{j}} .
$$

Dado $f \in \mathcal{F}(M)$, tal que $f>0, s \in \mathbb{R}, \operatorname{grad}\left(f^{s}\right)=s f^{s-1} \operatorname{grad} f$.

Definição A.11 O diferencial covariante de um campo vetorial $V$ em $M$ é o tensor $D V$ de ordem $(1,1)$ tal que, $\forall W \in \Re(M)$ e $\theta \in \mathfrak{X}^{*}(M)$,

$$
(D V)(\theta, W)=\left(D_{W} V\right)(\theta) .
$$

Proposição A.12 Seja $V$ um campo vetorial em $M$. O divergente de $V$ é definido como a contração do diferencial covariante de $V$. Se $\left\{E_{i}\right\}$ é um referencial ortonormal móvel, então,

$$
\operatorname{div}(V)=\sum_{i=1}^{n} g\left(E_{i}, E_{i}\right) g\left(D_{E_{i}} V, E_{i}\right) .
$$

Proposição A.13 O Hessiano de uma função $f \in \mathcal{F}(M)$, definido como

$$
H^{f}(X, Y)=X Y f-\left(D_{X} Y\right) f=g\left(D_{X} \operatorname{grad} f, Y\right),
$$

$\forall X, Y \in \mathfrak{X}(M)$, é um campo tensorial simétrico de ordem $(0,2)$.

Proposição A.14 $O$ Laplaciano de uma função $f \in \mathcal{F}(M)$ é o divergente do seu gradiente, isto é, $\Delta f=\operatorname{div}(\operatorname{grad} f)$, o que equivale a dizer que

$$
\Delta f=\sum_{i, j=1}^{n} g^{i j} H_{i j}^{f}, \text { onde } H_{i j}^{f}=H^{f}\left(E_{i}, E_{j}\right)
$$

$e\left\{E_{i}\right\}$ é uma base arbitrária de $T_{p} M$, onde $p \in M$ é arbitrário. Em um sistema de coordenadas locais,

$$
\Delta f=\sum_{i, j=1}^{n} g^{i j}\left(\frac{\partial^{2} f}{\partial x^{i} \partial x^{j}}-\sum_{k=1}^{n} \Gamma_{i j}^{k} \frac{\partial f}{\partial x^{k}}\right) .
$$


Segue que, dado $f \in \mathcal{F}(M)$, tal que $f>0, s \in \mathbb{R}$,

$$
\Delta\left(f^{s}\right)=s\left[(s-1) f^{s-2} g(\operatorname{grad} f, \operatorname{grad} f)+f^{s-1} \Delta f\right] .
$$

Proposição A.15 Considere duas variedades semi-Riemannianas $M$ e $N$ e uma homotetia $\psi$ entre elas. Isto é, um difeomorfismo $\psi: M \rightarrow N$ tal que $\psi^{*}\left(g_{N}\right)=$ $c\left(g_{M}\right)$, para algum $c \in \mathbb{R}, c \neq 0$, onde $g_{N}$ e $g_{M}$ indicam as métricas em $N$ e $M$, respectivamente. Então, a conexão de Levi-Civita é preservada. Isto é,

$$
\mathrm{d} \psi\left(D_{X}^{M} Y\right)=D_{\mathrm{d} \psi(X)}^{N} \mathrm{~d} \psi(Y), \forall X, Y \in \mathscr{X}(M)
$$

onde $D^{M}$ e $D^{N}$ indicam as conexões de Levi-Civita em $M$ e $N$, respectivamente. Além disso, o tensor de Riemann e o tensor de Ricci são, também, preservados. Entretanto, para a curvatura escalar, $S^{N} \circ \psi=\frac{1}{c} S^{M}$, onde $S^{N}$ e $S^{M}$ indicam as curvaturas escalares em $N$ e $M$, respectivamente. Note que uma isometria corresponde ao caso $c=1$.

Proposição A.16 Considere uma variedade semi-Riemanniana $\bar{M}$, uma subvariedade $M$ e $j: M \rightarrow \bar{M}$ a aplicação de inclusão, $M \subset \bar{M}$. Se o "pull-back" $j^{*}\left(g_{\bar{M}}\right)$ é um tensor métrico em $M,\left(M, j^{*}\left(g_{\bar{M}}\right)\right)$ é denominada uma subvariedade semiRiemanniana de $\bar{M}$. Neste caso, considere a decomposição

$$
T_{p}(\bar{M})=T_{p}(M) \oplus\left[T_{p}(M)\right]^{\perp}, \forall p \in M
$$

Com esta identificação, é válido que $D_{V}^{M} W=\operatorname{Tan}\left(D_{V}^{\bar{M}} W\right), \forall V, W \in \mathscr{X}(M)$, onde $D^{M}$ e $D^{\bar{M}}$ são as conexões de Levi-Civita em $M$ e $\bar{M}$, respectivamente e "Tan" indica a projeção de $T_{p}(\bar{M})$ em $T_{p}(M)$.

Proposição A.17 Nas condições descritas na proposição anterior, defina a segunda forma fundamental:

$$
I I: \mathscr{t}(M) \times \mathscr{X}(M) \rightarrow \mathscr{X}(M)^{\perp}
$$


por $I I(V, W)=\operatorname{Nor} D_{V}^{\bar{M}} W$, onde "Nor" indica a projeção de $T_{p}(\bar{M})$ em $\left[T_{p}(M)\right]^{\perp}$, $\forall V, W \in \mathscr{X}(M), p \in M$. Sejam, então, $R^{M}$ e $R^{\bar{M}}$ os tensores de Riemann em $M$ e $\bar{M}$, respectivamente e $g$ a métrica em $\bar{M}$. Neste caso,

$$
\begin{aligned}
g\left(R^{M}(V, W) X, Y\right)= & g\left(R^{\bar{M}}(V, W) X, Y\right)-g(I I(V, X), I I(W, Y)) \\
& +g(I I(V, Y), I I(W, X)),
\end{aligned}
$$

$\forall V, W, X, Y \in \mathscr{X}(M)$. Tal equação é denominada equação de Gauss.

Proposição A.18 Sejam $M$ uma variedade semi-Riemanniana, $p \in M$ e $A$ um tensor de ordem $(r, s)$ em $M$. Sejam $\hat{\theta}^{1}, \ldots, \hat{\theta}^{r}$ e $\theta^{1}, \ldots, \theta^{r}$ 1-formas tais que $\hat{\theta}^{i}(p)=$ $\theta^{i}(p), \forall i \in\{1, \ldots, r\}$; sejam $\hat{X}_{1}, \ldots, \hat{X}_{s}$ e $X_{1}, \ldots, X_{s}$ campos vetoriais tais que $\hat{X}_{i}(p)=X_{i}(p), \forall i \in\{1, \ldots, s\}$. Então

$$
\left[A\left(\hat{\theta}^{1}, \ldots, \hat{\theta}^{r}, \hat{X}_{1}, \ldots, \hat{X}_{s}\right)\right](p)=\left[A\left(\theta^{1}, \ldots, \theta^{r}, X_{1}, \ldots, X_{s}\right)\right](p) .
$$

Conseqüentemente, uma aplicação $A_{p}:\left(T_{p} M^{*}\right)^{r} \times\left(T_{p} M\right)^{s} \rightarrow \mathbb{R}$ é definida na forma que se segue: se $\alpha^{1}, \ldots, \alpha^{r} \in\left(T_{p} M\right)^{*}$ e $x_{1}, \ldots, x_{s} \in T_{p} M$,

$$
A_{p}\left(\alpha^{1}, \ldots, \alpha^{r}, x_{1}, \ldots, x_{s}\right)=\left[A\left(\theta^{1}, \ldots, \theta^{r}, X_{1}, \ldots, X_{s}\right)\right](p)
$$

onde $\theta^{1}, \ldots, \theta^{r}$ são quaisquer 1 -formas em $M$ tais que $\left.\theta^{i}\right|_{p}=\alpha^{i}, \forall i \in\{1, \ldots, r\}$ e $X_{1}, \ldots, X_{s}$ são quaisquer campos vetoriais em $M$ tais que $\left.X_{i}\right|_{p}=x_{i}, \forall i \in\{1, \ldots, s\}$.

Proposição A.19 Considere B uma variedade semi-Riemanniana arbitrária, tal que $\operatorname{dim}(B)=1$. Neste caso,

$$
\begin{aligned}
& R^{B}(X, Y) Z=0, \\
& \operatorname{Ric}^{B}(X, Y)=0, \forall X, Y, Z \in \nVdash(B) \quad e \\
& S^{B}=0 .
\end{aligned}
$$




\section{Referências Bibliográficas}

[1] L. J. Alías, A. Romero e M. Sánchez, Spacelike hypersurfaces of constant mean curvature and Calabi-Bernstein type problems, Tôhoku Math. J., 49, 1997, 337-345.

[2] J. P. Baker, Twice Warped Products, M. Sc. Thesis, University of MissouriColumbia, Columbia, MO, 1997.

[3] M. Bañados, C. Teitelboim e J. Zanelli, The blackhole in three dimensional spacetime, Phys. Rev. Letters, 69, 1992, 1849-1851.

[4] J. K. Beem e P. E. Ehrlich, Global Lorentzian Geometry, New York, 1.ed., 1981, Marcel-Dekker Inc.

[5] J. K. Beem, P. E. Ehrlich e K. L. Easley, Global Lorentzian Geometry, New York, 2.ed., 1996, Marcel-Dekker Inc.

[6] J. K. Beem e T. G. Powell, Geodesic completeness and maximality in Lorentzian Warped Products, Tensor (NS), 39, 1982, 31-36.

[7] A. L. Besse, Einstein Manifolds, Springer-Verlag, Germany, 1987.

[8] R. L. Bishop e B. O'Neill, Manifolds of negative curvature, Trans. Amer. Math. Soc., 145, 1969, 1-49.

[9] M. Brozos-Vázquez, E. García-Río e R. Vázquez-Lorenzo, Locally conformally flat multidimensional cosmological models with a higher-dimensional external space-time, Class. Quantum Grav., 22, 2005, 3119-3133.

[10] J. Choi, Multiply warped products with non smooth metrics, J. Math. Phys., 41, 2000, 8163-8169.

[11] F. Dobarro e L. Dozo, Scalar curvature and warped products of Riemann manifolds, Trans. Amer. Math. Soc., 303, 1987, 161-168. 
[12] F. Dobarro e B. Ünal, Curvature in special base conformal warped products, Math. DG/0412436, 2004.

[13] F. Dobarro e B. Ünal, Curvature of multiply warped products, J. Geom. Phys., 55, 2005, 75-106.

[14] P. E. Ehrlich, Y. Jung e S. Kim, Constant scalar curvatures on warped product manifolds, Tsukuba J. Math., 20, 1996, 239-256.

[15] S. W. Hawking e G. F. R. Ellis, The Large Scale Structure of Space-Time, Cambridge University Press, U. K., 1973.

[16] S. Hong, J. Choi e Y. Park, (2+1) BTZ black hole and multiply warped product spacetimes, Gen. Relativity Gravitation, 35, 2003, 2105-2116.

[17] M. Ito, Warped geometry in higher dimensions with an orbifold extra dimensions, Phys. Rev. D, 64, 2001.

[18] E. Kasner, Geometrical theorems in Einstein's cosmological equations, Amer. J. of Math., 43, 1921, 217.

[19] S. S. Kokarev, Multidimensional generalization of Kasner solution, Grav. Cosmol., 2, 1996, 321-324.

[20] D. Kramer, E. Herlt, M. MacCallum e H. Stephani, Exact solutions of Einstein's Field Equations, Cambridge University Press, U. K., 1980.

[21] C. W. Misner, K. S. Thorne e J. A. Wheeler, Gravitation, Freeman and Company, San Francisco, 1973.

[22] B. O'Neill, Semi-Riemannian Geometry, Academic Press, New York, 1983.

[23] R. K. Sachs e H. Wu, General Relativity for Mathematicians, Springer-Verlag, New York, 1977.

[24] M. Sánchez, On the geometry of generalized Robertson-Walker space-times: geodesics, Gen. Relativity Gravitation, 30, 1998, 915-932.

[25] B. Ünal, Doubly Warped Products, Ph. D. Thesis, University of MissouriColumbia, 2000.

[26] B. Ünal, Multiply warped products, J. Geom. Phys., 34, 2000, 287-301.

[27] B. Ünal, Doubly warped products, Dif. Geom. Appl., 15, 2001, 253-263. 